\title{
On lattice models of gapped phases with fusion category symmetries
}

\author{
Kansei Inamura \\ Institue for Solid State Physics, University of Tokyo, \\ Kashiwa, Chiba 277-8581, Japan \\ E-mail: k_inamura@issp.u-tokyo.ac.jp
}

ABSTRACT: We construct topological quantum field theories (TQFTs) and commuting projector Hamiltonians for any $1+1 \mathrm{~d}$ gapped phases with non-anomalous fusion category symmetries, i.e. finite symmetries that admit SPT phases. The construction is based on two-dimensional state sum TQFT whose input datum is an $H$-simple left $H$-comodule algebra, where $H$ is a finite dimensional semisimple Hopf algebra. We show that the actions of fusion category symmetries $\mathcal{C}$ on the boundary conditions of these state sum TQFTs are represented by module categories over $\mathcal{C}$. This agrees with the classification of gapped phases with symmetry $\mathcal{C}$. We also find that the commuting projector Hamiltonians for these state sum TQFTs have fusion category symmetries at the level of the lattice models and hence provide lattice realizations of gapped phases with fusion category symmetries. As an application, we discuss the edge modes of SPT phases based on these commuting projector Hamiltonians. Finally, we mention that we can extend the construction of topological field theories to the case of anomalous fusion category symmetries by replacing a semisimple Hopf algebra with a semisimple pseudo-unitary connected weak Hopf algebra.

KEywords: Discrete Symmetries, Global Symmetries, Lattice Quantum Field Theory, Topological Field Theories

ARXIV EPRINT: 2110.12882 


\section{Contents}

1 Introduction and summary 1

2 Preliminaries $\quad 5$

2.1 Fusion categories, tensor functors, and module categories 5

2.2 Hopf algebras, (co)module algebras, and smash product 9

2.3 Representation categories of Hopf algebras 11

3 Pullback of fusion category TQFTs by tensor functors 13

$\begin{array}{lll}3.1 & \text { TQFTs with fusion category symmetries } & 13\end{array}$

$\begin{array}{lll}3.2 & \text { Pullback of TQFTs by tensor functors } & 15\end{array}$

4 State sum TQFTs and commuting projector Hamiltonians 17

$\begin{array}{lll}4.1 & \text { State sum TQFTs with defects } & 17\end{array}$

$\begin{array}{lll}4.2 & \text { Pullback of state sum TQFTs } & 22\end{array}$

4.3 Commuting projector Hamiltonians 24

4.4 Examples: gapped phases of finite gauge theories 26

4.5 Edge modes of SPT phases with fusion category symmetries 28

$\begin{array}{lll}4.6 & \text { Generalization to anomalous fusion category symmetries } & 28\end{array}$

$\begin{array}{ll}\text { A State sum TQFTs on surfaces with interfaces } & 29\end{array}$

\section{Introduction and summary}

Symmetries of physical systems are characterized by the algebraic relations of topological defects. For instance, ordinary group symmetries are associated with invertible topological defects with codimension one. When the codimensions of invertible topological defects are greater than one, the corresponding symmetries are called higher form symmetries [1]. We can generalize these symmetries by relaxing the invertibility of topological defects. Symmetries associated with such non-invertible topological defects are called non-invertible symmetries, which are studied recently in various contexts [2-42]. The algebraic structures of non-invertible symmetries are in general captured by higher categories [40-44]. In particular, non-invertible symmetries associated with finitely many topological defect lines in $1+1$ dimensions are described by unitary fusion categories [13]. These symmetries are called fusion category symmetries [15] and are investigated extensively [8-31].

Fusion category symmetries are ubiquitous in two-dimensional conformal field theories (CFTs). A basic example is the symmetry of the Ising CFT [27, 45, 46]: the Ising CFT has a fusion category symmetry generated by the non-invertible Kramers-Wannier duality defect 
and the invertible $\mathbb{Z}_{2}$ spin-flip defect. ${ }^{1}$ More generally, any diagonal RCFTs have fusion category symmetries generated by the Verlinde lines [48]. Fusion category symmetries are also studied in other CFTs such as CFTs with central charge $c=1[20,23,49]$ and RCFTs that are not necessarily diagonal $[50-56]^{2}$

We can also consider fusion category symmetries in topological quantum field theories (TQFTs). In particular, it is shown in $[15,19]$ that unitary TQFTs with fusion category symmetry $\mathcal{C}$ are classified by semisimple module categories over $\mathcal{C}$. This result will be heavily used in the rest of this paper. This classification reveals that fusion category symmetries do not always admit SPT phases, i.e. symmetric gapped phases with unique ground states. If fusion category symmetries do not have SPT phases, they are said to be anomalous [15], and otherwise non-anomalous.

Fusion category symmetries exist on the lattice as well. Remarkably, 2d statistical mechanical models with general fusion category symmetries are constructed recently in [27, 28]. There are also examples of $1+1 \mathrm{~d}$ lattice models known as anyonic chains [29-31]. These models might cover all the gapped phases with fusion category symmetries. However, to the best of my knowledge, systematic construction of $1+1 \mathrm{~d}$ TQFTs and corresponding gapped Hamiltonians with fusion category symmetries is still lacking.

In this paper, we explicitly construct TQFTs and commuting projector Hamiltonians for any $1+1 d$ gapped phases with arbitrary non-anomalous fusion category symmetries. For this purpose, we first show that a TQFT with fusion category symmetry, which is formulated axiomatically in [13], is obtained from another TQFT with different symmetry by a procedure that we call pullback. This is a natural generalization of the pullback of an SPT phase with finite group symmetry by a group homomorphism [57]. Specifically, we can pull back topological defects of a TQFT with symmetry $\mathcal{C}^{\prime}$ by a tensor functor $F: \mathcal{C} \rightarrow \mathcal{C}^{\prime}$ to obtain a TQFT with symmetry $\mathcal{C}$. This corresponds to the fact that given a $\mathcal{C}^{\prime}$-module category $\mathcal{M}$ and a tensor functor $F: \mathcal{C} \rightarrow \mathcal{C}^{\prime}$, we can endow $\mathcal{M}$ with a $\mathcal{C}$-module category structure. By using this technique, we can construct any TQFTs with non-anomalous fusion category symmetries. ${ }^{3}$ To see this, we first recall that nonanomalous symmetries are described by fusion categories that admit fiber functors [15, $19,22]$. Such fusion categories are equivalent to the representation categories $\operatorname{Rep}(H)$ of finite dimensional semisimple Hopf algebras $H$. Therefore, TQFTs with non-anomalous fusion category symmetries are classified by semisimple module categories over $\operatorname{Rep}(H)$. Among these module categories, we are only interested in indecomposable ones because any semisimple module category can be decomposed into a direct sum of indecomposable module categories. Every indecomposable semisimple module category over $\operatorname{Rep}(H)$ is equivalent to the category ${ }_{K} \mathcal{M}$ of left $K$-modules where $K$ is an $H$-simple left $H$-comodule

\footnotetext{
${ }^{1}$ The fusion category that describes this symmetry is known as a $\mathbb{Z}_{2}$ Tambara-Yamagami category [13$17,47]$.

${ }^{2}$ Precisely, $c=1$ CFTs can have infinitely many topological defect lines labeled by continuous parameters $[20,23,49]$, whose algebraic structure should be described by a mathematical framework beyond fusion categories.

${ }^{3}$ We can also construct any TQFTs with anomalous fusion category symmetries in the same way, see section 4.6 .
} 
algebra [58]. The $\operatorname{Rep}(H)$-module category structure on ${ }_{K} \mathcal{M}$ is represented by a tensor functor from $\operatorname{Rep}(H)$ to the category $\operatorname{End}\left({ }_{K} \mathcal{M}\right)$ of endofunctors of ${ }_{K} \mathcal{M}$. Since $\operatorname{End}\left({ }_{K} \mathcal{M}\right)$ is equivalent to the category ${ }_{K} \mathcal{M}_{K}$ of $K-K$ bimodules [59], we have a tensor functor $F_{K}: \operatorname{Rep}(H) \rightarrow{ }_{K} \mathcal{M}_{K}$. We can use this tensor functor to pull back a ${ }_{K} \mathcal{M}_{K}$ symmetric TQFT to a $\operatorname{Rep}(H)$ symmetric TQFT. We show in section 3 that a $\operatorname{Rep}(H)$ symmetric TQFT corresponding to a $\operatorname{Rep}(H)$-module category ${ }_{K} \mathcal{M}$ is obtained as the pullback of a specific ${ }_{K} \mathcal{M}_{K}$ symmetric TQFT, which corresponds to the same category ${ }_{K} \mathcal{M}$ regarded as a ${ }_{K} \mathcal{M}_{K}$-module category, by a tensor functor $F_{K}$.

We also describe the pullback in the context of state sum TQFTs in section 4. Here, a state sum TQFT is a TQFT obtained by state sum construction [60], which is a recipe to construct a $2 \mathrm{~d}$ TQFT from a semisimple algebra. The pullback of the state sum TQFTs enables us to explicitly construct TQFTs with $\operatorname{Rep}(H)$ symmetry. Specifically, it turns out that the state sum TQFT obtained from an $H$-simple left $H$-comodule algebra $K$ is a $\operatorname{Rep}(H)$ symmetric TQFT ${ }_{K} \mathcal{M}$. Any indecomposable TQFTs with $\operatorname{Rep}(H)$ symmetry can be constructed in this way because an $H$-simple left $H$-comodule algebra is always semisimple [61].

The existence of the state sum construction suggests that we can realize the $\operatorname{Rep}(H)$ symmetric TQFTs by lattice models. Indeed, the vacua of a state sum TQFT are in one-to-one correspondence with the ground states of an appropriate commuting projector Hamiltonian [62,63]. Specifically, when the input algebra of a state sum TQFT is $K$, the commuting projector Hamiltonian $H$ is given by

$$
H=\sum_{i}\left(1-h_{i, i+1}\right), \quad h_{i, i+1}=\Delta_{K} \circ m_{K}=\overbrace{K_{i}}^{K_{i}} \overbrace{\Delta_{K}}^{K_{i+1}}: K_{i} \otimes K_{i+1} \rightarrow K_{i} \otimes K_{i+1}
$$

where $K_{i}:=K$ is the local Hilbert space on the lattice, $m_{K}: K \otimes K \rightarrow K$ is multiplication on $K$, and $\Delta_{K}: K \rightarrow K \otimes K$ is comultiplication for the Frobenius algebra structure on $K$. The diagram in the above equation is the string diagram representation of the linear map $h_{i, i+1}$. We find that when $K$ is a left $H$-comodule algebra, we can define the action of $\operatorname{Rep}(H)$ on the lattice Hilbert space $\mathcal{H}=\bigotimes_{i} K_{i}$ via the left $H$-comodule action on $K$. Here, we need to choose $K$ appropriately so that the $\operatorname{Rep}(H)$ action becomes faithful on the lattice. In section 4 , we show that the above Hamiltonian has a $\operatorname{Rep}(H)$ symmetry by explicitly computing the commutation relation of the Hamiltonian (1.1) and the action of the $\operatorname{Rep}(H)$ symmetry. Moreover, we will see that the $\operatorname{Rep}(H)$ symmetry action of the lattice model agrees with that of the state sum TQFT when the Hilbert space $\mathcal{H}$ is restricted to the subspace spanned by the ground states. This implies that the commuting projector Hamiltonian (1.1) realizes a $\operatorname{Rep}(H)$ symmetric TQFT ${ }_{K} \mathcal{M}$.

We also examine the edge modes of SPT phases with $\operatorname{Rep}(H)$ symmetry by putting the systems on an interval. The ground states of the commuting projector Hamiltonian (1.1) on an interval are described by the input algebra $K$ itself $[64,65]$. In particular, for SPT phases, $K$ is isomorphic to the endomorphism algebra $\operatorname{End}(M) \cong M^{*} \otimes M$ of a simple left $K$-module $M$. We can interpret $M^{*}$ and $M$ as the edge modes by using the matrix product 
state (MPS) representation of the ground states. Thus, the edge modes of the Hamiltonian (1.1) for a $\operatorname{Rep}(H)$ SPT phase ${ }_{K} \mathcal{M}$ become either a left $K$-module $M$ or a right $K$ module $M^{*}$ depending on which boundary they are localized to. As a special case, we reproduce the well-known result that the edge modes of an SPT phase with finite group symmetry $G$ have anomalies, which take values in the second group cohomology $H^{2}(G, \mathrm{U}(1))$. We note that the edge modes of the Hamiltonian (1.1) are not necessarily minimal: it would be possible to partially lift the degeneracy on the boundaries by adding symmetric perturbations.

Although we will only consider the fixed point Hamiltonians (1.1) in this paper, we can add terms to our models while preserving the $\operatorname{Rep}(H)$ symmetry. In general, the lattice models still have the $\operatorname{Rep}(H)$ symmetry if the additional terms are $H$-comodule maps. Since the Hamiltonians with additional terms are generically no longer exactly solvable, one would use numerical calculations to determine the phase diagrams. For this purpose, we need to write the Hamiltonians in the form of matrices by choosing a basis of the lattice Hilbert space $\mathcal{H}$. As a concrete example, we will explicitly compute the action of the Hamiltonian (1.1) with $\operatorname{Rep}(G)$ symmetry by choosing a specific basis of $\mathcal{H}$. Here, $\operatorname{Rep}(G)$ is the category of representations of a finite group $G$, which describes the symmetry of $G$ gauge theory.

Before proceeding to the next section, we comment on a relation between the state sum models discussed in this paper and the anyon chain models. ${ }^{4}$ As we summarized above, we construct a $\operatorname{Rep}(H)$ symmetric commuting projector Hamiltonian of the state sum model by using a left $H$-comodule algebra $K$ in this paper. On the other hand, we can also construct a $\operatorname{Rep}\left(\left(H^{*}\right)^{\mathrm{cop}}\right)$ symmetric commuting projector Hamiltonian of the anyon chain model by using the same algebra $K{ }^{5}$ where $\left(H^{*}\right)^{\text {cop }}$ is the coopposite coalgebra of the dual Hopf algebra $H^{*}$. The anyon chain with $\operatorname{Rep}\left(\left(H^{*}\right)^{\mathrm{cop}}\right)$ symmetry is a lattice model whose Hilbert space is spanned by fusion trees in $\operatorname{Rep}\left(\left(H^{*}\right)^{\mathrm{cop}}\right)$. The commuting projector Hamiltonian of the anyon chain can be written diagrammatically as

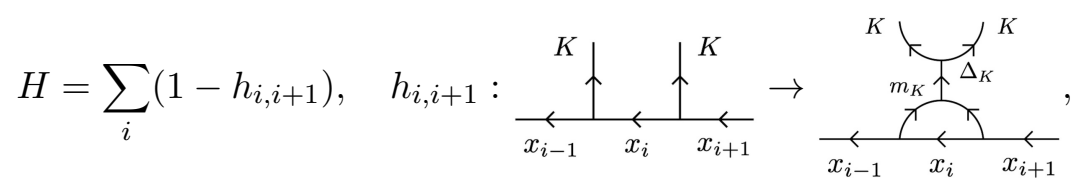

where the horizontal edges of the fusion diagrams are labeled by objects in $\operatorname{Rep}\left(\left(H^{*}\right)^{\mathrm{cop}}\right)$. We note that a left $H$-comodule algebra $K$ is an algebra object in $\operatorname{Rep}\left(\left(H^{*}\right)^{\text {cop }}\right)$. The right diagram in eq. (1.2) can be deformed to a sum of fusion trees via $F$-moves and hence the Hamiltonian can be explicitly written in terms of $F$-symbols. The above Hamiltonian has ground states represented by the fusion trees all of whose horizontal edges are labeled by a right $K$-module $M \in \operatorname{Rep}\left(\left(H^{*}\right)^{\mathrm{cop}}\right)$. This suggests, though not prove, that the gapped phase of this anyon chain corresponds to the category of right $K$-modules in $\operatorname{Rep}\left(\left(H^{*}\right)^{\mathrm{cop}}\right)$, which is a $\operatorname{Rep}\left(\left(H^{*}\right)^{\mathrm{cop}}\right)$-module category. As we will argue in section 4.1 , this also suggests that the gapped phase of the anyon chain model constructed from the opposite algebra $K^{\mathrm{op}}$ is obtained by the generalized gauging of the state sum model constructed from $K$, see footnote 14 .

\footnotetext{
${ }^{4}$ The author thanks Kantaro Ohmori for a discussion on the anyon chain models.

${ }^{5}$ Equivalently, a $\operatorname{Rep}(H)$ symmetric commuting projector Hamiltonian of the anyon chain model is obtained from a left $H$-module algebra instead of a left $H$-comodule algebra.
} 
The reason why the state sum model (1.1) and the anyon chain model (1.2) have different symmetries despite the similarity between their Hamiltonians is that the symmetry actions are defined differently due to the different structures of their Hilbert spaces. Specifically, the $\operatorname{Rep}\left(\left(H^{*}\right)^{\mathrm{cop}}\right)$ symmetry of the anyon chain model is defined via the fusion of topological defect lines and the horizontal edges, whereas the $\operatorname{Rep}(H)$ symmetry of the state sum model is defined via the $H$-comodule structure on the algebra $K$ as we will discuss in section 4, see eq. (4.24). Since the state sum models do not have counterparts of horizontal edges of fusion trees, the $\operatorname{Rep}\left(\left(H^{*}\right)^{\text {cop }}\right)$ symmetry does not act on the state sum models. Conversely, since the $\operatorname{Rep}(H)$ action (4.24) is not a morphism in $\operatorname{Rep}\left(\left(H^{*}\right)^{\mathrm{cop}}\right)$ and therefore is not given by a fusion diagram in $\operatorname{Rep}\left(\left(H^{*}\right)^{\mathrm{cop}}\right)$, the $\operatorname{Rep}(H)$ symmetry does not act on the anyon chains.

The rest of the paper is organized as follows. In section 2 , we briefly review some mathematical backgrounds. In section 3, we introduce the notion of pullback of a TQFT and show that every TQFT with non-anomalous fusion category symmetry $\operatorname{Rep}(H)$ is obtained by pulling back a ${ }_{K} \mathcal{M}_{K}$ symmetric TQFT ${ }_{K} \mathcal{M}$ by a tensor functor $F_{K}: \operatorname{Rep}(H) \rightarrow{ }_{K} \mathcal{M}_{K}$. In section 4 , we define state sum TQFTs with $\operatorname{Rep}(H)$ symmetry and show that they are realized by the commuting projector Hamiltonians (1.1). We emphasize that these Hamiltonians have fusion category symmetries at the level of the lattice models. These lattice realizations enable us to examine the edge modes of $\operatorname{Rep}(H)$ SPT phases. We also comment on a generalization to TQFTs and commuting projector Hamiltonians with anomalous fusion category symmetries in the last subsection. In appendix A, we describe state sum TQFTs with fusion category symmetries in the presence of interfaces.

\section{Preliminaries}

\subsection{Fusion categories, tensor functors, and module categories}

We begin with a brief review of unitary fusion categories, tensor functors, and module categories [66]. A unitary fusion category $\mathcal{C}$ is equipped with a bifunctor $\otimes: \mathcal{C} \times \mathcal{C} \rightarrow \mathcal{C}$, which is called a tensor product. The tensor product of objects $x, y \in \mathcal{C}$ is denoted by $x \otimes y$. The tensor product $(x \otimes y) \otimes z$ of three objects $x, y, z \in \mathcal{C}$ is related to $x \otimes(y \otimes z)$ by a natural isomorphism $\alpha_{x, y, z}:(x \otimes y) \otimes z \rightarrow x \otimes(y \otimes z)$ called an associator, which satisfies the following pentagon equation:

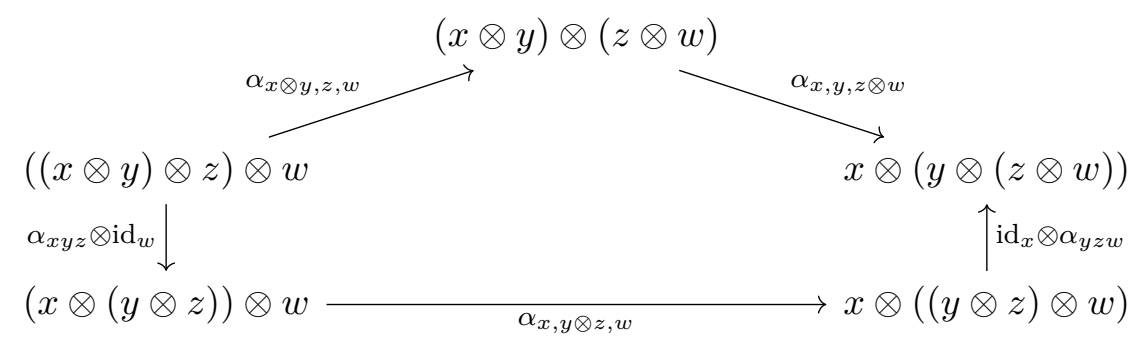

There is a unit object $1 \in \mathcal{C}$ that behaves as a unit of the tensor product, i.e. $1 \otimes x \cong x \otimes 1 \cong$ $x$. The isomorphisms $l_{x}: 1 \otimes x \rightarrow x$ and $r_{x}: x \otimes 1 \rightarrow x$ are called a left unit morphism and 
a right unit morphism respectively. These isomorphisms satisfy the following commutative diagram:

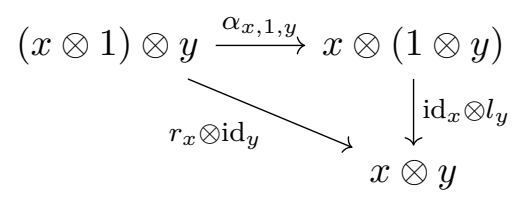

We can always take $l_{x}$ and $r_{x}$ as the identity morphism $\operatorname{id}_{x}$ by identifying $1 \otimes x$ and $x \otimes 1$ with $x$. In sections 3 and 4 , we assume $l_{x}=r_{x}=\mathrm{id}_{x}$.

A unitary fusion category $\mathcal{C}$ also has an additive operation $\oplus: \mathcal{C} \times \mathcal{C} \rightarrow \mathcal{C}$ called a direct sum. An object $x \in \mathcal{C}$ is called a simple object when it cannot be decomposed into a direct sum of other objects. In particular, the unit object $1 \in \mathcal{C}$ is simple. The number of (isomorphism classes of) simple objects is finite, and every object is isomorphic to a direct sum of finitely many simple objects. Namely, for any object $x \in \mathcal{C}$, we have an isomorphism $x \cong \bigoplus_{i} N_{i} a_{i}$ where $\left\{a_{i}\right\}$ is a set of simple objects and $N_{i}$ is a non-negative integer.

The Hom space $\operatorname{Hom}(x, y)$ for any objects $x, y \in \mathcal{C}$ is a finite dimensional $\mathbb{C}$-vector space equipped with an adjoint $\dagger: f \in \operatorname{Hom}(x, y) \mapsto f^{\dagger} \in \operatorname{Hom}(y, x)$. The associators, the left unit morphisms, and the right unit morphisms are unitary with respect to this adjoint, i.e. $\alpha_{x, y, z}^{\dagger}=\alpha_{x, y, z}^{-1}, l_{x}^{\dagger}=l_{x}^{-1}$, and $r_{x}^{\dagger}=r_{x}^{-1}$. We note that the endomorphism space of a simple object $a_{i}$ is one-dimensional, i.e. $\operatorname{End}\left(a_{i}\right):=\operatorname{Hom}\left(a_{i}, a_{i}\right) \cong \mathbb{C}$.

For every object $x \in \mathcal{C}$, we have a dual object $x^{*} \in \mathcal{C}$ and a pair of morphisms $\mathrm{ev}_{x}^{L}: x^{*} \otimes x \rightarrow 1$ and $\operatorname{coev}_{x}^{L}: 1 \rightarrow x \otimes x^{*}$ that satisfy the following relations:

$$
\begin{gathered}
r_{x} \circ\left(\operatorname{id}_{x} \otimes \mathrm{ev}_{x}^{L}\right) \circ \alpha_{x, x^{*}, x} \circ\left(\operatorname{coev}_{x}^{L} \otimes \mathrm{id}_{x}\right) \circ l_{x}^{-1}=\mathrm{id}_{x}, \\
l_{x^{*}} \circ\left(\mathrm{ev}_{x}^{L} \otimes \mathrm{id}_{x^{*}}\right) \circ \alpha_{x^{*}, x, x^{*}}^{-1} \circ\left(\operatorname{id}_{x^{*}} \otimes \operatorname{coev}_{x}^{L}\right) \circ r_{x^{*}}^{-1}=\operatorname{id}_{x^{*}} .
\end{gathered}
$$

These morphisms are called left evaluation and left coevaluation morphisms respectively. The adjoints of these morphisms $\mathrm{ev}_{x}^{R}:=\left(\operatorname{coev}_{x}^{L}\right)^{\dagger}$ and $\operatorname{coev}_{x}^{R}:=\left(\mathrm{ev}_{x}^{L}\right)^{\dagger}$ are called right evaluation and right coevaluation morphisms, which satisfy similar relations to eqs. (2.3) and (2.4).

A tensor functor $F: \mathcal{C} \rightarrow \mathcal{C}^{\prime}$ between fusion categories $\mathcal{C}$ and $\mathcal{C}^{\prime}$ is a functor equipped with a natural isomorphism $J_{x, y}: F(x) \otimes F(y) \rightarrow F(x \otimes y)$ and an isomorphism $\phi: 1^{\prime} \rightarrow$ $F(1)$ that satisfy the following commutative diagrams:

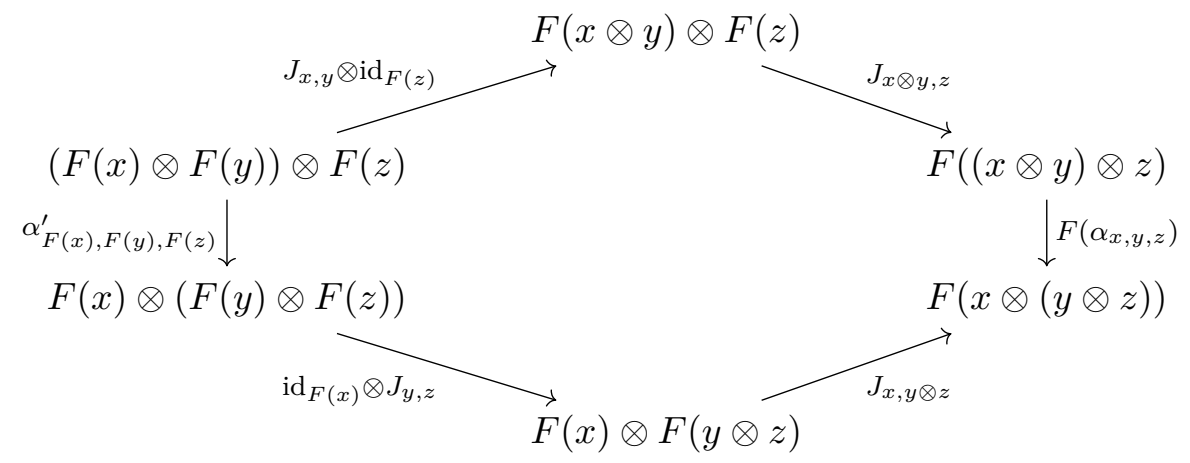




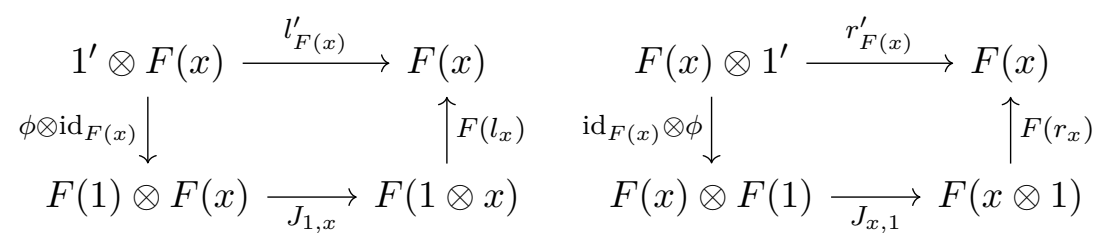

Here, 1 and $1^{\prime}$ are unit objects of $\mathcal{C}$ and $\mathcal{C}^{\prime}$ respectively. When $\mathcal{C}$ and $\mathcal{C}^{\prime}$ are unitary fusion categories, we require that $J_{x, y}$ and $\phi$ are unitary in the sense that $J_{x, y}^{\dagger}=J_{x, y}^{-1}$ and $\phi^{\dagger}=\phi^{-1}$. The isomorphism $\phi$ can always be chosen as the identity morphism by the identification $1^{\prime}=F(1)$.

A module category $\mathcal{M}$ over a fusion category $\mathcal{C}$ is a category equipped with a bifunctor $\bar{\otimes}: \mathcal{C} \times \mathcal{M} \rightarrow \mathcal{M}$, which represents the action of $\mathcal{C}$ on $\mathcal{M}$. For any objects $x, y \in \mathcal{C}$ and $M \in \mathcal{M}$, we have a natural isomorphism $m_{x, y, M}:(x \otimes y) \bar{\otimes} M \rightarrow x \bar{\otimes}(y \bar{\otimes} M)$ called a module associativity constraint that satisfies the following commutative diagram:

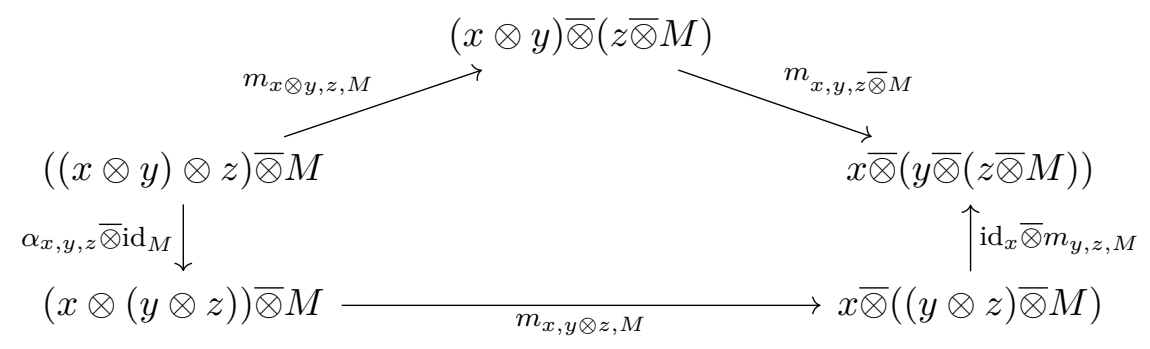

The action of the unit object $1 \in \mathcal{C}$ gives an isomorphism $l_{M}: 1 \bar{\otimes} M \rightarrow M$ called a unit constraint such that the following diagram commutes:

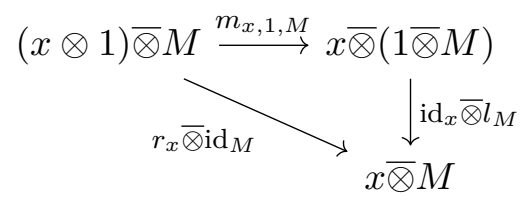

A $\mathcal{C}$-module category structure on $\mathcal{M}$ can also be represented by a tensor functor from $C$ to the category of endofunctors of $M$, i.e. $F: \mathcal{C} \rightarrow \operatorname{End}(\mathcal{M})$, which is analogous to an action of an algebra on a module. A module category $\mathcal{M}$ is said to be indecomposable if it cannot be decomposed into a direct sum of two non-trivial module categories.

When we have a tensor functor $(F, J, \phi): \mathcal{C} \rightarrow \mathcal{C}^{\prime}$, we can regard a $\mathcal{C}^{\prime}$-module category $M$ as a $\mathcal{C}$-module category by defining the action of $\mathcal{C}$ on $\mathcal{M}$ as $x \bar{\otimes} M:=F(x) \bar{\otimes}^{\prime} M$ for $x \in \mathcal{C}$ and $M \in \mathcal{M}$, where $\bar{\otimes}^{\prime}$ is the action of $\mathcal{C}^{\prime}$ on $\mathcal{M}$. The natural isomorphisms $m_{x, y, M}$ and $l_{M}$ are given by

$$
m_{x, y, M}=m_{F(x), F(y), M}^{\prime} \circ\left(J_{x, y}^{-1} \bar{\otimes}^{\prime} \mathrm{id}_{M}\right), \quad l_{M}=l_{M}^{\prime} \circ\left(\phi^{-1} \bar{\otimes}^{\prime} \mathrm{id}_{M}\right),
$$

where $m^{\prime}$ and $l^{\prime}$ are the module associativity constraint and the unit constraint for the $\mathcal{C}^{\prime}$-module category structure on $\mathcal{M}$.

An important example of a unitary fusion category is the category ${ }_{K} \mathcal{M}_{K}$ of $K-K$ bimodules where $K$ is a finite dimensional semisimple algebra. We review this category 
in some detail for later convenience. The objects and morphisms of ${ }_{K} \mathcal{M}_{K}$ are $K-K$ bimodules and $K-K$ bimodule maps respectively. The monoidal structure on ${ }_{K} \mathcal{M}_{K}$ is given by the tensor product over $K$, which is usually denoted by $\otimes_{K}$. To describe the tensor product $Y_{1} \otimes_{K} Y_{2}$ of $K-K$ bimodules $Y_{1}, Y_{2} \in{ }_{K} \mathcal{M}_{K}$, we first recall that a finite dimensional semisimple algebra $K$ is a Frobenius algebra. Here, an algebra $K$ equipped with multiplication $m_{K}: K \otimes K \rightarrow K$ and a unit $\eta_{K}: \mathbb{C} \rightarrow K$ is called a Frobenius algebra if it is also a coalgebra equipped with comultiplication $\Delta_{K}: K \rightarrow K \otimes K$ and a counit $\epsilon_{K}: K \rightarrow \mathbb{C}$ such that the following Frobenius relation is satisfied:

$$
\left(m_{K} \otimes \operatorname{id}_{K}\right) \circ\left(\operatorname{id}_{K} \otimes \Delta_{K}\right)=\left(\operatorname{id}_{K} \otimes m_{K}\right) \circ\left(\Delta_{K} \otimes \operatorname{id}_{K}\right)=\Delta_{K} \circ m_{K} .
$$

In the string diagram notation, the above relation is represented as

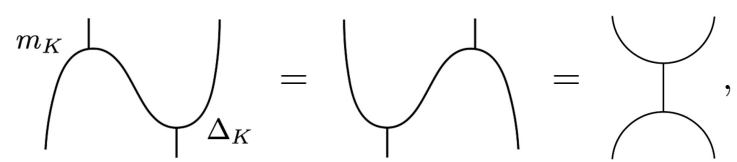

where each string and junction represent the algebra $K$ and the (co)multiplication respectively. In our convention, we read these diagrams from bottom to top. The comultiplication $\Delta_{K}$ and the counit $\epsilon_{K}$ can be written in terms of the multiplication $m_{K}$ and the unit $\eta_{K}$ as follows [67]:

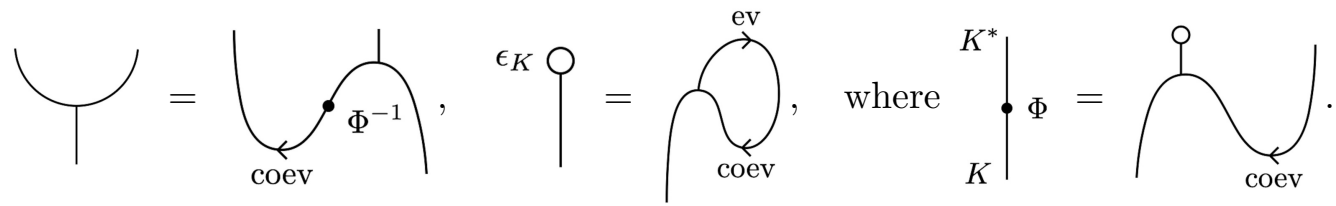

In the above equation, $K^{*}$ denotes the dual vector space of $K$. The linear maps ev and coev are the evaluation and coevaluation morphisms of the category of vector spaces. Specifically, we have

$$
\operatorname{ev}(a \otimes f)=f(a), \quad \operatorname{coev}(\lambda)=\lambda \sum_{i} u_{i} \otimes u^{i}, \quad \forall a \in K, \forall f \in K^{*}, \forall \lambda \in \mathbb{C},
$$

where $\left\{u_{i}\right\}$ and $\left\{u^{i}\right\}$ are dual bases of $K$ and $K^{*}$. It turns out that the Frobenius algebra structure given by eq. (2.12) satisfies the following two properties [52]:6

$\Delta$-separability:

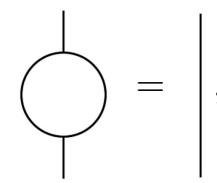

symmetricity:
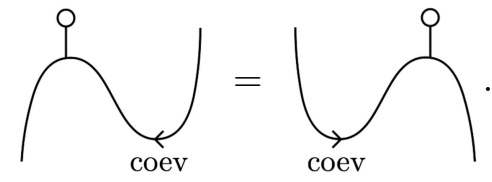

The tensor product $Y_{1} \otimes_{K} Y_{2}$ is defined as the image of a projector $p_{Y_{1}, Y_{2}}: Y_{1} \otimes Y_{2} \rightarrow Y_{1} \otimes Y_{2}$ that is represented by the following string diagram

$$
p_{Y_{1}, Y_{2}}=\left.\left.\underbrace{}_{Y_{1}}\right|_{\eta_{K}}\right|_{Y_{2}},
$$

\footnotetext{
${ }^{6}$ In general, given a $\Delta$-separable symmetric Frobenius algebra object $K$ in a fusion category $\mathcal{C}$, the category of $K-K$ bimodules in $\mathcal{C}$ also becomes a fusion category. The category ${ }_{K} \mathcal{M}_{K}$ is a special case where $\mathcal{C}$ is the category of vector spaces.
} 
where the junction of $Y_{1}\left(Y_{2}\right)$ and $K$ represents a right (left) $K$-module action. We note that the unit object for the tensor product over $K$ is $K$ itself. The splitting maps of the projector (2.15) are denoted by $\pi_{Y_{1}, Y_{2}}: Y_{1} \otimes Y_{2} \rightarrow Y_{1} \otimes_{K} Y_{2}$ and $\iota_{Y_{1}, Y_{2}}: Y_{1} \otimes_{K} Y_{2} \rightarrow Y_{1} \otimes Y_{2}$, which obey $\iota_{Y_{1}, Y_{2}} \circ \pi_{Y_{1}, Y_{2}}=p_{Y_{1}, Y_{2}}$ and $\pi_{Y_{1}, Y_{2}} \circ \iota_{Y_{1}, Y_{2}}=\operatorname{id}_{Y_{1} \otimes_{K} Y_{2}}$. The associator $\alpha_{Y_{1}, Y_{2}, Y_{3}}$ : $\left(Y_{1} \otimes_{K} Y_{2}\right) \otimes_{K} Y_{3} \rightarrow Y_{1} \otimes_{K}\left(Y_{2} \otimes_{K} Y_{3}\right)$ is given by a composition of these splitting maps as

$$
\alpha_{Y_{1}, Y_{2}, Y_{3}}=\pi_{Y_{1}, Y_{2} \otimes_{K} Y_{3}} \circ\left(\operatorname{id}_{Y_{1}} \otimes \pi_{Y_{2}, Y_{3}}\right) \circ\left(\iota_{Y_{1}, Y_{2}} \otimes \operatorname{id}_{Y_{3}}\right) \circ \iota_{Y_{1} \otimes_{K} Y_{2}, Y_{3}} .
$$

The tensor product of morphisms $f \in \operatorname{Hom}_{K K}\left(Y_{1}, Y_{1}^{\prime}\right)$ and $g \in \operatorname{Hom}_{K K}\left(Y_{2}, Y_{2}^{\prime}\right)$ is defined in terms of the splitting maps as $f \otimes_{K} g:=\pi_{Y_{1}, Y_{2}} \circ(f \otimes g) \circ \iota_{Y_{1}, Y_{2}}$, where $\operatorname{Hom}_{K K}\left(Y, Y^{\prime}\right)$ denotes the space of $K-K$ bimodule maps from $Y$ to $Y^{\prime}$.

We finally notice that the category ${ }_{K} \mathcal{M}$ of left $K$-modules is a ${ }_{K} \mathcal{M}_{K}$-module category, on which ${ }_{K} \mathcal{M}_{K}$ acts by the tensor product over $K$. The module associativity constraint $m_{Y_{1}, Y_{2}, M}:\left(Y_{1} \otimes_{K} Y_{2}\right) \otimes_{K} M \rightarrow Y_{1} \otimes_{K}\left(Y_{2} \otimes_{K} M\right)$ for $Y_{1}, Y_{2} \in{ }_{K} \mathcal{M}_{K}$ and $M \in{ }_{K} \mathcal{M}$ is given by the composition of the splitting maps as the associator (2.16):

$$
m_{Y_{1}, Y_{2}, M}=\pi_{Y_{1}, Y_{2} \otimes_{K} M} \circ\left(\operatorname{id}_{Y_{1}} \otimes \pi_{Y_{2}, M}\right) \circ\left(\iota_{Y_{1}, Y_{2}} \otimes \operatorname{id}_{M}\right) \circ \iota_{Y_{1} \otimes_{K} Y_{2}, M} .
$$

\subsection{Hopf algebras, (co)module algebras, and smash product}

In this subsection, we briefly review the definitions and some basic properties of Hopf algebras. For details, see for example [68-70]. We first give the definition. A $\mathbb{C}$-vector space $H$ is called a Hopf algebra if it is equipped with structure maps $(m, 1, \Delta, \epsilon, S)$ that satisfy the following conditions:

1. $(H, m, 1)$ is a unital associative algebra where $m: H \otimes H \rightarrow H$ is the multiplication and $1 \in H$ is the unit.

2. $(H, \Delta, \epsilon)$ is a counital coassociative coalgebra where $\Delta: H \rightarrow H \otimes H$ is the comultiplication and $\epsilon: H \rightarrow \mathbb{C}$ is the counit. ${ }^{7}$

3. The comultiplication $\Delta$ is a unit-preserving algebra homomorphism

$$
\Delta(g h)=\Delta(g) \Delta(h), \quad \Delta(1)=1 \otimes 1, \quad \forall g, h \in H,
$$

where we denote the multiplication of $g$ and $h$ as $g h$. The multiplication on $H \otimes H$ is induced by that on $H$.

4. The counit $\epsilon$ is a unit-preserving algebra homomorphism ${ }^{8}$

$$
\epsilon(g h)=\epsilon(g) \epsilon(h), \quad \epsilon(1)=1, \quad \forall g, h \in H .
$$

5. The antipode $S: H \rightarrow H$ satisfies

$$
m \circ(\mathrm{id} \otimes S) \circ \Delta=m \circ(S \otimes \mathrm{id}) \circ \Delta=1 \epsilon .
$$

\footnotetext{
${ }^{7}$ The comultiplication $\Delta$ for the Hopf algebra structure on a semisimple Hopf algebra $H$ is different from the comultiplication $\Delta_{H}$ for the Frobenius algebra structure on $H$. The same comment applies to $\epsilon$ and $\epsilon_{H}$.

${ }^{8}$ The right-hand side of the second equation of (2.19) is just a number $1 \in \mathbb{C}$, which defers from the unit of $H$.
} 
In particular, the antipode $S$ squares to the identity when $H$ is semisimple, i.e. $S^{2}=$ id. In the rest of this paper, we only consider finite dimensional semisimple Hopf algebras and do not distinguish between $S$ and $S^{-1}$.

When $H$ is a Hopf algebra, the opposite algebra $H^{\text {op }}$ is also a Hopf algebra, whose underlying vector space is $H$ and whose structure maps are given by $\left(m^{\mathrm{op}}, 1, \Delta, \epsilon, S^{-1}\right)$. Here, the opposite multiplication $m^{\mathrm{op}}: H^{\mathrm{op}} \otimes H^{\mathrm{op}} \rightarrow H^{\mathrm{op}}$ is defined by $m^{\mathrm{op}}(g \otimes h)=h g$ for all $g, h \in H$. Similarly, the coopposite coalgebra $H^{\text {cop }}$ also becomes a Hopf algebra, whose underlying vector space is $H$ and whose structure maps are given by $\left(m, 1, \Delta^{\text {cop }}, \epsilon, S^{-1}\right)$.

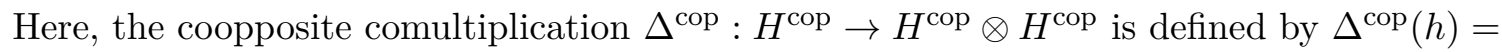
$h_{(2)} \otimes h_{(1)}$ for all $h \in H .^{9}$

In the subsequent sections, we will use the string diagram notation where the above conditions $1-5$ are represented as follows:

1.

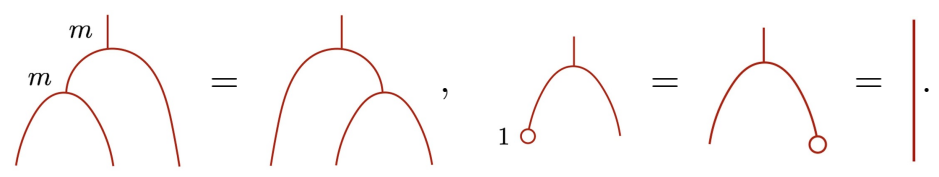

2 .

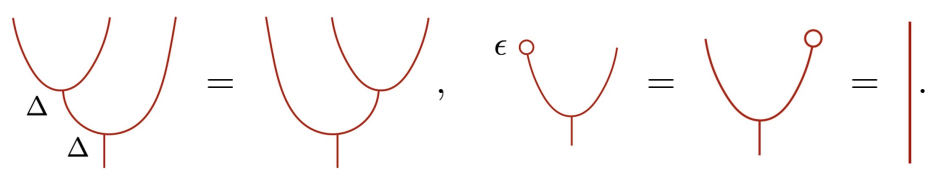

3.

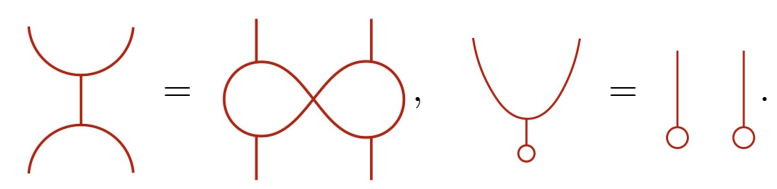

4.

$$
P=9 \uparrow, \quad 9=1 .
$$

5.

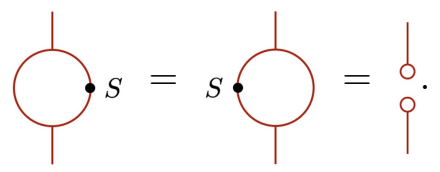

A left $H$-module $A$ is a vector space on which $H$ acts from the left. The $H$-module action $\rho_{A}: H \otimes A \rightarrow A$ is a linear map that satisfies
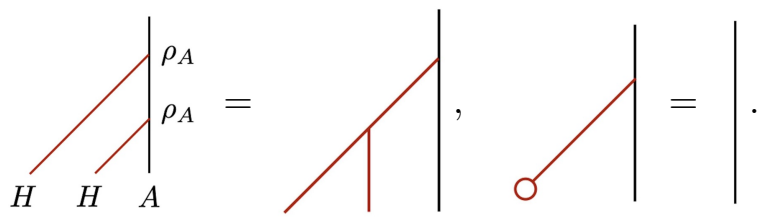

\footnotetext{
${ }^{9}$ We use Sweedler's notation for the comultiplication $\Delta(h)=h_{(1)} \otimes h_{(2)}$.
} 
When a left $H$-module $A$ has an algebra structure that is compatible with the $H$-module structure, $A$ is called a left $H$-module algebra. More precisely, a left $H$-module $A$ with a module action $\rho_{A}: H \otimes A \rightarrow A$ is a left $H$-module algebra if $\left(A, m_{A}, \eta_{A}\right)$ is a unital associative algebra such that

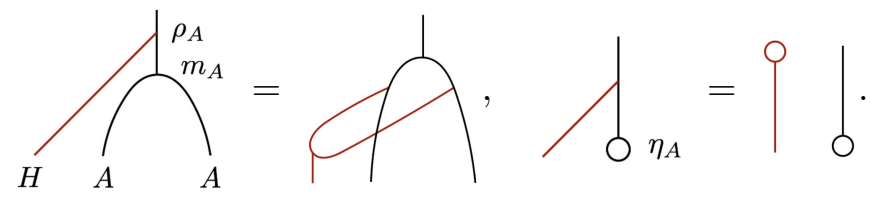

A left $H$-module algebra $A$ is said to be $H$-simple if $A$ does not have any proper non-zero ideal $I$ such that $\rho_{A}(H \otimes I) \subset I$.

We can also define a left $H$-comodule algebra similarly. A left $H$-comodule algebra $K$ is a unital associative algebra whose algebra structure $\left(K, m_{K}, \eta_{K}\right)$ is compatible with the $H$-comodule action $\lambda_{K}: K \rightarrow H \otimes K$ in the following sense:

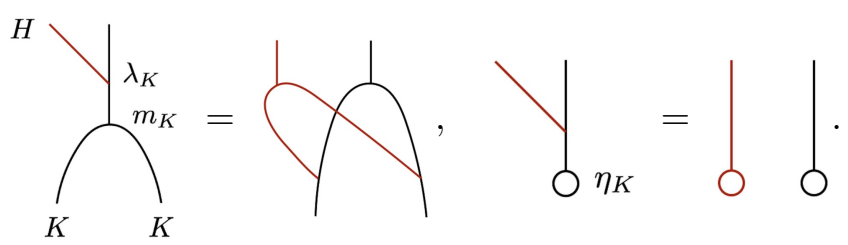

A left $H$-comodule algebra $K$ is said to be $H$-simple if $K$ does not have any proper non-zero ideal $I$ such that $\lambda_{K}(I) \subset H \otimes I$. In particular, an $H$-simple left $H$-comodule algebra $K$ is semisimple [61]. The left $H$-comodule action on $K$ is said to be inner-faithful if there is no proper Hopf subalgebra $H^{\prime} \subset H$ such that $\lambda_{K}(K) \subset H^{\prime} \otimes K[71]$.

Given a left $H$-module algebra $A$, we can construct a left $H^{\text {cop }}$-comodule algebra $A \# H$ called the smash product of $A$ and $H$. As a vector space, $A \# H$ is the same as the tensor product $A \otimes H$. The left $H^{\mathrm{cop}}$-comodule action on $A \# H$ is defined via the coopposite comultiplication $\Delta^{\mathrm{cop}}$ as

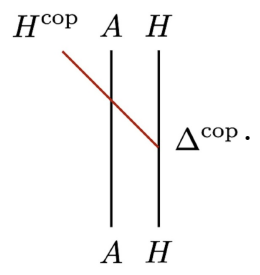

The algebra structure on $A \# H$ is given by

$$
(a \# h) \cdot\left(a^{\prime} \# h^{\prime}\right)=a\left(h_{(1)} \cdot a^{\prime}\right) \# h_{(2)} h^{\prime}, \quad \forall a, a^{\prime} \in A, \forall h, h^{\prime} \in H
$$

\subsection{Representation categories of Hopf algebras}

Every non-anomalous fusion category symmetry is equivalent to the representation category of a Hopf algebra. ${ }^{10}$ In this subsection, we describe the representation category of a Hopf algebra and module categories over it following [58].

\footnotetext{
${ }^{10}$ We recall that fusion category symmetries are said to be non-anomalous if and only if they admit SPT phases, i.e. gapped phases with unique ground states [15].
} 
The representation category $\operatorname{Rep}(H)$ of a Hopf algebra $H$ is a category whose objects are left $H$-modules and whose morphisms are left $H$-module maps. The tensor product $V \otimes W$ of left $H$-modules $V$ and $W$ is given by the usual tensor product over $\mathbb{C}$. The left $H$ module structure on the tensor product $V \otimes W$ is defined via the comultiplication $\Delta$. Specifically, if we denote the left $H$-module action on $V \in \operatorname{Rep}(H)$ as $\rho_{V}: H \otimes V \rightarrow V$, we have

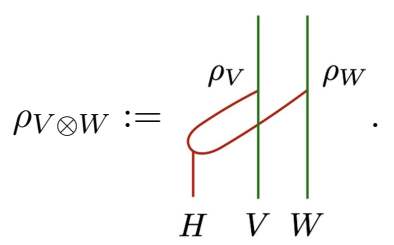

When $V \in \operatorname{Rep}(H)$ is a left $H$-module, the dual vector space $V^{*}$ is also a left $H$-module with the left $H$-module action given by

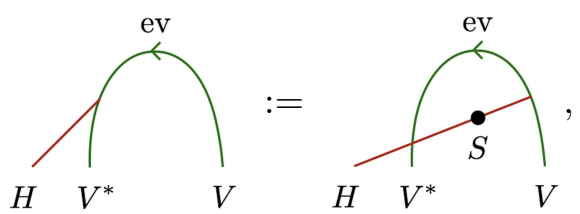

where ev $: V^{*} \otimes V \rightarrow \mathbb{C}$ is a usual paring defined by $\operatorname{ev}(\varphi \otimes v):=\varphi(v)$ for $\varphi \in V^{*}, v \in V$.

An indecomposable semisimple module category over $\operatorname{Rep}(H)$ is equivalent to the category of right $A$-modules in $\operatorname{Rep}(H)$ where $A$ is an $H$-simple left $H$-module algebra [72, 73]. We denote this module category as $\left({ }_{H} \mathcal{M}\right)_{A}$. As a module category over $\operatorname{Rep}(H)$, the category $\left({ }_{H} \mathcal{M}\right)_{A}$ is equivalent to the category of left $A^{\mathrm{op}} \# H^{\mathrm{cop}}$-modules [58], which we denote by $A^{\mathrm{op}} \# H^{\mathrm{cop}} \mathcal{M}$ :

$$
\left({ }_{H} \mathcal{M}\right)_{A} \cong{ }_{A^{\mathrm{op}} \# H^{\mathrm{cop}} \mathcal{M}}
$$

We note that $A^{\mathrm{op}}$ is a left $H^{\mathrm{cop}}$-module algebra when $A$ is a left $H$-module algebra, and hence $A^{\mathrm{op}} \# H^{\mathrm{cop}}$ is a left $H$-comodule algebra. Moreover, when $A$ is $H$-simple as a module algebra, $A^{\mathrm{op}} \# H^{\mathrm{cop}}$ is also $H$-simple as a comodule algebra. Therefore, every indecomposable semisimple module category over $\operatorname{Rep}(H)$ is equivalent to the category of left modules over an $H$-simple left $H$-comodule algebra $A^{\text {op }} \# H^{\text {cop }}$. Conversely, for any $H$-simple left $H$-comodule algebra $K$, the category ${ }_{K} \mathcal{M}$ of left $K$-modules becomes an indecomposable semisimple module category over $\operatorname{Rep}(H)$ [58]. The action of $\operatorname{Rep}(H)$ on ${ }_{K} \mathcal{M}$ is given by the usual tensor product, i.e. $V \bar{\otimes} M:=V \otimes M$ for $V \in \operatorname{Rep}(H)$ and $M \in_{K} \mathcal{M}$. The left $K$-module structure on $V \bar{\otimes} M$ is defined via the $H$-comodule structure on $K$ as

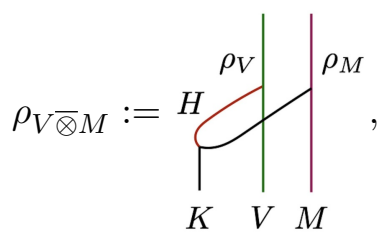

where $\rho_{M}: K \otimes M \rightarrow M$ is the left $K$-module action on $M$. We note that the $\operatorname{Rep}(H)$ module category structure on ${ }_{K} \mathcal{M}$ is represented by a tensor functor from $\operatorname{Rep}(H)$ to the category $\operatorname{End}\left({ }_{K} \mathcal{M}\right)$ of endofunctors of ${ }_{K} \mathcal{M}$. Since the category $\operatorname{End}\left({ }_{K} \mathcal{M}\right)$ is equivalent to 


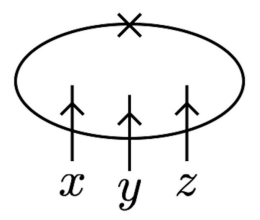

Figure 1. The Hilbert space on the above spatial circle is given by $Z((x \otimes y) \otimes z)$, where the base point is represented by the cross mark in the above figure. We can also assign a Hilbert space to a circle with an arbitrary number of topological defects in a similar way.
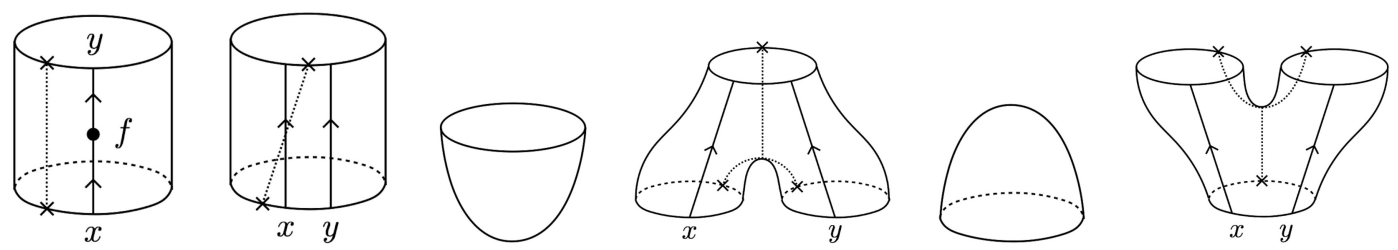

Figure 2. The building blocks of linear maps: a cylinder amplitude $Z(f)$, a change of the case point $X_{x, y}$, a unit $\eta$, multiplication $M_{x, y}$, a counit $\epsilon$, and comultiplication $\Delta_{x, y}$ (from left to right). Each diagram represents a linear map from the Hilbert space assigned to the bottom to the Hilbert space assigned to the top.

the category of $K-K$ bimodules, we have a tensor functor $F_{K}: \operatorname{Rep}(H) \rightarrow{ }_{K} \mathcal{M}_{K}$, which maps an object $V \in \operatorname{Rep}(H)$ to a $K-K$ bimodule $F_{K}(V)=V \otimes K$. This tensor functor induces a $\operatorname{Rep}(H)$-module category structure on a ${ }_{K} \mathcal{M}_{K}$-module category ${ }_{K} \mathcal{M}$ via eq. (2.9).

\section{$3 \quad$ Pullback of fusion category TQFTs by tensor functors}

In this section, we show that given a $2 \mathrm{~d}$ TQFT $Q^{\prime}$ with symmetry $\mathcal{C}^{\prime}$ and a tensor functor $F: \mathcal{C} \rightarrow \mathcal{C}^{\prime}$, we can construct a 2 d TQFT $Q$ with symmetry $\mathcal{C}$ by pulling back the TQFT $Q^{\prime}$ by the tensor functor $F$. In particular, we can construct any 2d TQFT with non-anomalous fusion category symmetry $\operatorname{Rep}(H)$ by pulling back a specific ${ }_{K} \mathcal{M}_{K}$ symmetric TQFT by a tensor functor $F_{K}: \operatorname{Rep}(H) \rightarrow{ }_{K} \mathcal{M}_{K}$. We note that the content of this section can also be applied to anomalous fusion category symmetries as well as non-anomalous ones.

\subsection{TQFTs with fusion category symmetries}

We first review the axiomatic formulation of $2 \mathrm{~d}$ unitary TQFT with fusion category symmetry $\mathcal{C}$ following [13]. A $2 \mathrm{~d}$ TQFT assigns a Hilbert space $Z(x)$ to a spatial circle that has a topological defect $x \in \mathcal{C}$ running along the time direction. When the spatial circle has multiple topological defects $x, y, z, \cdots$, the Hilbert space is given by $Z(((x \otimes y) \otimes z) \otimes \cdots)$, where the order of the tensor product is determined by the position of the base point on the circle, see figure 1.

A 2d TQFT also assigns a linear map to a two-dimensional surface decorated by a network of topological defects. The linear map assigned to an arbitrary surface is composed of the following building blocks, see also figure 2 : 
1. a cylinder amplitude $Z(f): Z(x) \rightarrow Z(y), \quad \forall f \in \operatorname{Hom}(x, y)$,

2. a change of the base point $X_{x, y}: Z(x \otimes y) \rightarrow Z(y \otimes x)$,

3. a unit $\eta: \mathbb{C} \rightarrow Z(1)$,

4. multiplication $M_{x, y}: Z(x) \otimes Z(y) \rightarrow Z(x \otimes y)$,

5. a counit $\epsilon: Z(1) \rightarrow \mathbb{C}$,

6. comultiplication $\Delta_{x, y}: Z(x \otimes y) \rightarrow Z(x) \otimes Z(y)$.

For unitary TQFTs, the counit $\epsilon$ and the comultiplication $\Delta_{x, y}$ are the adjoints of the unit $\eta$ and the multiplication $M_{x, y}$ respectively, i.e. $\epsilon=\eta^{\dagger}$ and $\Delta_{x, y}=M_{x, y}^{\dagger}$. In particular, the counit $\epsilon$ and the comultiplication $\Delta_{x, y}$ are no longer independent data of a TQFT.

For the well-definedness of the cylinder amplitude, we require that $Z(f)$ is $\mathbb{C}$-linear in morphisms and preserves the composition of morphisms:

$$
\begin{aligned}
Z\left(\lambda f+\lambda^{\prime} f^{\prime}\right) & =\lambda Z(f)+\lambda^{\prime} Z\left(f^{\prime}\right), & \forall \lambda, \lambda^{\prime} \in \mathbb{C}, \forall f, f^{\prime} \in \operatorname{Hom}(x, y), \\
Z(g \circ f) & =Z(g) \circ Z(f) & \forall f \in \operatorname{Hom}(x, y), \forall g \in \operatorname{Hom}(y, z) .
\end{aligned}
$$

Thus, a 2d TQFT with fusion category symmetry $\mathcal{C}$ gives a functor $Z: \mathcal{C} \rightarrow$ Vec from $\mathcal{C}$ to the category of vector spaces. This functor obeys various consistency conditions so that the assignment of Hilbert spaces and linear maps are well-defined. Specifically, a TQFT with fusion category symmetry $\mathcal{C}$ is a functor $Z: \mathcal{C} \rightarrow$ Vec equipped with a set of linear maps $(X, \eta, M)$ that satisfies the following consistency conditions [13]:

1. Well-definedness of the change of the base point:

$$
X_{x, y}=X_{y, x}^{-1}
$$

2. Naturality of the change of the base point:

$$
\begin{array}{ll}
Z\left(\operatorname{id}_{y} \otimes f\right) \circ X_{x, y}=X_{x^{\prime}, y} \circ Z\left(f \otimes \mathrm{id}_{y}\right) & \forall f \in \operatorname{Hom}\left(x, x^{\prime}\right), \\
Z\left(g \otimes \operatorname{id}_{x}\right) \circ X_{x, y}=X_{x, y^{\prime}} \circ Z\left(\operatorname{id}_{x} \otimes g\right) & \forall g \in \operatorname{Hom}\left(y, y^{\prime}\right) .
\end{array}
$$

3. Associativity of the change of the base point:

$$
X_{y, z \otimes x} \circ Z\left(\alpha_{y z x}\right) \circ X_{x, y \otimes z} \circ Z\left(\alpha_{x y z}\right)=Z\left(\alpha_{z x y}^{-1}\right) \circ X_{x \otimes y, z} .
$$

4. Non-degeneracy of the pairing:

The pairing $\eta^{\dagger} \circ Z\left(\mathrm{ev}_{x}^{L}\right) \circ M_{x^{*}, x}: Z\left(x^{*}\right) \otimes Z(x) \rightarrow \mathbb{C}$ is non-degenerate.

5. Unit constraint:

$$
M_{1, x} \circ\left(\eta \otimes \operatorname{id}_{Z(x)}\right)=\operatorname{id}_{Z(x)}=M_{x, 1} \circ\left(\operatorname{id}_{Z(x)} \otimes \eta\right) .
$$


6. Associativity of the multiplication:

$$
Z\left(\alpha_{x y z}\right) \circ M_{x \otimes y, z} \circ\left(M_{x, y} \otimes \operatorname{id}_{Z(z)}\right)=M_{x, y \otimes z} \circ\left(\operatorname{id}_{Z(x)} \otimes M_{y, z}\right) .
$$

7. Twisted commutativity:

$$
M_{y, x}\left(\psi_{y} \otimes \psi_{x}\right)=X_{x, y} \circ M_{x, y}\left(\psi_{x} \otimes \psi_{y}\right) \quad \forall \psi_{x} \in Z(x), \forall \psi_{y} \in Z(y) .
$$

8. Naturality of the multiplication:

$$
\begin{aligned}
& M_{x^{\prime}, y} \circ\left(Z(f) \otimes \operatorname{id}_{Z(y)}\right)=Z\left(f \otimes \operatorname{id}_{y}\right) \circ M_{x, y}, \\
& M_{x, y^{\prime}} \circ\left(\operatorname{id}_{Z(x)} \otimes Z(g)\right)=Z\left(\operatorname{id}_{x} \otimes g\right) \circ M_{x, y} .
\end{aligned}
$$

9. Uniqueness of the multiplication:

$$
\begin{aligned}
& Z\left(\left(\operatorname{id}_{x} \otimes \mathrm{ev}_{z}^{L}\right) \otimes \operatorname{id}_{y^{*}}\right) \circ \mathcal{A}_{\left(x \otimes z^{*}\right) \otimes\left(z \otimes y^{*}\right) \rightarrow\left(x \otimes\left(z^{*} \otimes z\right)\right) \otimes y^{*}} \circ M_{x \otimes z^{*}, z \otimes y^{*}} \\
& =Z\left(\mathrm{id}_{x \otimes y^{*}} \otimes \mathrm{ev}_{z}^{R}\right) \circ \mathcal{A}_{\left(z^{*} \otimes x\right) \otimes\left(y^{*} \otimes z\right) \rightarrow\left(x \otimes y^{*}\right) \otimes\left(z \otimes z^{*}\right)} \circ M_{z^{*} \otimes x, y^{*} \otimes z} \circ\left(X_{x, z^{*}} \otimes X_{z, y^{*}}\right),
\end{aligned}
$$

where $\mathcal{A}$ is a generalized associator that we will define below.

10. Consistency on the torus:

$$
\begin{aligned}
& \mathcal{A}_{(x \otimes w) \otimes(z \otimes y) \rightarrow(y \otimes x) \otimes(w \otimes z)} \circ M_{x \otimes w, z \otimes y} \circ\left(X_{w, x} \otimes X_{y, z}\right) \circ M_{w \otimes x, y \otimes z}^{\dagger} \\
& =M_{y \otimes x, w \otimes z} \circ\left(X_{x, y} \otimes X_{z, w}\right) \circ M_{x \otimes y, z \otimes w}^{\dagger} \circ \mathcal{A}_{(w \otimes x) \otimes(y \otimes z) \rightarrow(x \otimes y) \otimes(z \otimes w)} .
\end{aligned}
$$

In the last two equations, the generalized associator $\mathcal{A}_{p \rightarrow q}: Z(p) \rightarrow Z(q)$ is defined as a composition of the change of the base point $X$ and the associator $Z(\alpha)$. We note that the isomorphism $\mathcal{A}_{p \rightarrow q}$ is uniquely determined by $p$ and $q$ [13].

In summary, a $2 \mathrm{~d}$ unitary TQFT with fusion category symmetry $\mathcal{C}$ is a functor $Z$ : $\mathcal{C} \rightarrow$ Vec equipped with a triple $(X, \eta, M)$ that satisfies the consistency conditions (3.3)$(3.12)$. It is shown in $[15,19]$ that 2 d unitary TQFTs with fusion category symmetry $\mathcal{C}$ are classified by semisimple module categories over $\mathcal{C}$. Namely, each 2 d unitary TQFT with symmetry $\mathcal{C}$ is labeled by a semisimple $\mathcal{C}$-module category. The TQFT labeled by a $\mathcal{C}$-module category $\mathcal{M}$ has the category of boundary conditions described by $\mathcal{M}[19,74]$, whose semisimplicity follows from the unitarity of the TQFT $[74,75]$.

\subsection{Pullback of TQFTs by tensor functors}

Let $\left(Z^{\prime}, X^{\prime}, \eta^{\prime}, M^{\prime}\right)$ be a 2 d TQFT with symmetry $\mathcal{C}^{\prime}$. Given a tensor functor $(F, J, \phi)$ : $\mathcal{C} \rightarrow \mathcal{C}^{\prime}$, we can construct a $2 \mathrm{~d}$ TQFT $(Z, X, \eta, M)$ with symmetry $\mathcal{C}$ as follows: the functor $Z: \mathcal{C} \rightarrow$ Vec is given by the composition $Z:=Z^{\prime} \circ F$, and the linear maps $(X, \eta, M)$ are defined as

$$
\begin{aligned}
X_{x, y} & :=Z^{\prime}\left(J_{y, x}\right) \circ X_{F(x), F(y)}^{\prime} \circ Z^{\prime}\left(J_{x, y}^{-1}\right), \\
\eta & :=Z^{\prime}(\phi) \circ \eta^{\prime} \\
M_{x, y} & :=Z^{\prime}\left(J_{x, y}\right) \circ M_{F(x), F(y)}^{\prime} .
\end{aligned}
$$


We can show that the quadruple $(Z, X, \eta, M)$ defined as above becomes a $2 \mathrm{~d}$ TQFT, provided that $\left(Z^{\prime}, X^{\prime}, \eta^{\prime}, M^{\prime}\right)$ satisfies the consistency conditions (3.3)-(3.12). We will explicitly check some of the consistency conditions for $(Z, X, \eta, M)$ below. The other equations can also be checked similarly.

Let us begin with eq. (3.3). This equation holds because the right-hand side can be written as

$$
\left(Z^{\prime}\left(J_{x, y}\right) \circ X_{F(y), F(x)}^{\prime} \circ Z^{\prime}\left(J_{y, x}^{-1}\right)\right)^{-1}=Z^{\prime}\left(J_{y, x}\right) \circ X_{F(x), F(y)}^{\prime} \circ Z^{\prime}\left(J_{x, y}^{-1}\right)=(\text { l.h.s. }),
$$

where we used the fact that $X^{\prime}$ satisfies eq. (3.3). Equation (3.4) follows from the naturality of $J$ :

$$
F(g \otimes f) \circ J_{y, x}=J_{y^{\prime}, x^{\prime}} \circ(F(g) \otimes F(f)), \quad \forall g \in \operatorname{Hom}\left(y, y^{\prime}\right), \forall f \in \operatorname{Hom}\left(x, x^{\prime}\right) .
$$

Indeed, if we choose either $g$ or $f$ as the identity morphism and use eq. (3.4) for $X^{\prime}$, we obtain eq. (3.4) for $X$. To show eq. (3.5), we note that $F\left(\alpha_{x y z}\right)$ can be written in terms of the associators $\alpha_{F(x), F(y), F(z)}^{\prime}$ of $\mathcal{C}^{\prime}$ due to the commutative diagram (2.5) as follows:

$$
F\left(\alpha_{x y z}\right)=J_{x, y \otimes z} \circ\left(\operatorname{id}_{F(x)} \otimes J_{y, z}\right) \circ \alpha_{F(x), F(y), F(z)}^{\prime} \circ\left(J_{x, y}^{-1} \otimes \operatorname{id}_{F(z)}\right) \circ J_{x \otimes y, z}^{-1} .
$$

We also notice that the naturality (3.4) of $X^{\prime}$ implies

$$
\begin{aligned}
& X_{F(x \otimes y), F(z)}^{\prime}=Z^{\prime}\left(\operatorname{id}_{F(z)} \otimes J_{x, y}\right) \circ X_{F(x) \otimes F(y), F(z)}^{\prime} \circ Z^{\prime}\left(J_{x, y}^{-1} \otimes \operatorname{id}_{F(z)}\right), \\
& X_{F(x), F(y \otimes z)}^{\prime}=Z^{\prime}\left(J_{y, z} \otimes \operatorname{id}_{F(x)}\right) \circ X_{F(x), F(y) \otimes F(z)}^{\prime} \circ Z^{\prime}\left(\operatorname{id}_{F(x)} \otimes J_{y, z}^{-1}\right) .
\end{aligned}
$$

By plugging eqs. (3.18) and (3.19) into the left-hand side of eq. (3.5), we find

$$
\begin{aligned}
(\text { l.h.s. })= & Z^{\prime}\left(J_{z \otimes x, y}\right) \circ Z^{\prime}\left(J_{z, x} \otimes \operatorname{id}_{F(y)}\right) \circ X_{F(y), F(z) \otimes F(x)}^{\prime} \circ Z^{\prime}\left(\alpha_{F(y), F(z), F(x)}^{\prime}\right) \\
& \circ X_{F(x), F(y) \otimes F(z)}^{\prime} \circ Z^{\prime}\left(\alpha_{F(x), F(y), F(z)}^{\prime}\right) \circ Z^{\prime}\left(J_{x, y}^{-1} \otimes \operatorname{id}_{F(z)}\right) \circ Z^{\prime}\left(J_{x \otimes y, z}^{-1}\right) \\
= & Z^{\prime}\left(J_{z \otimes x, y}\right) \circ Z^{\prime}\left(J_{z, x} \otimes \operatorname{id}_{F(y)}\right) \circ Z^{\prime}\left(\left(\alpha_{F(z), F(x), F(y)}^{\prime}\right)^{-1}\right) \\
& \circ X_{F(x) \otimes F(y), F(z)}^{\prime} \circ Z^{\prime}\left(J_{x, y}^{-1} \otimes \operatorname{id}_{F(z)}\right) \circ Z^{\prime}\left(J_{x \otimes y, z}^{-1}\right) \\
= & (\text { r.h.s. }) .
\end{aligned}
$$

The non-degeneracy condition (3.6) for an object $x \in \mathcal{C}$ follows from that for $F(x) \in \mathcal{C}^{\prime}$ because

$$
\eta^{\dagger} \circ Z\left(\operatorname{ev}_{x}^{L}\right) \circ M_{x^{*}, x}=\left(\eta^{\prime}\right)^{\dagger} \circ Z^{\prime}\left(\operatorname{ev}_{F(x)}^{L}\right) \circ M_{F(x)^{*}, F(x)}^{\prime},
$$

where we used $F(x)^{*}=F\left(x^{*}\right)$ and $F\left(\operatorname{ev}_{x}^{L}\right)=\phi \circ \operatorname{ev}_{F(x)}^{L} \circ J_{x^{*}, x}^{-1}$, cf. Exercise 2.10.6. in [66]. The unit constraint (3.7) is an immediate consequence of the commutative diagram (2.6) and eqs. (3.7) and (3.10) for $\left(\eta^{\prime}, M^{\prime}\right)$.

We can also check the remaining equations similarly. Thus, we find that the quadruple $(Z, X, \eta, M)$ becomes a $2 \mathrm{~d}$ TQFT with symmetry $\mathcal{C}$. We call a TQFT $(Z, X, \eta, M)$ the pullback of a TQFT $\left(Z^{\prime}, X^{\prime}, \eta^{\prime}, M^{\prime}\right)$ by a tensor functor $(F, J, \phi)$. 
By using the pullback, we can construct all the TQFTs with non-anomalous fusion category symmetry $\mathcal{C} .{ }^{11}$ To see this, we first recall that every non-anomalous fusion category symmetry $\mathcal{C}$ is equivalent to the representation category $\operatorname{Rep}(H)$ of a Hopf algebra $H$. Indecomposable semisimple module categories over $\operatorname{Rep}(H)$ are given by the categories ${ }_{K} \mathcal{M}$ of left $K$-modules where $K$ is an $H$-simple left $H$-comodule algebra. Accordingly, we have a tensor functor $F_{K}: \operatorname{Rep}(H) \rightarrow{ }_{K} \mathcal{M}_{K}$ that represents the $\operatorname{Rep}(H)$-module category structure on ${ }_{K} \mathcal{M}$. Therefore, we can pull back a ${ }_{K} \mathcal{M}_{K}$ symmetric TQFT by $F_{K}$ to obtain a $\operatorname{Rep}(H)$ symmetric TQFT. Here, we notice that there is a canonical ${ }_{K} \mathcal{M}_{K}$ symmetric TQFT labeled by a ${ }_{K} \mathcal{M}_{K}$-module category ${ }_{K} \mathcal{M}$, whose module category structure was discussed in section 2.1. Thus, by pulling back this canonical ${ }_{K} \mathcal{M}_{K}$ symmetric TQFT ${ }_{K} \mathcal{M}$ by the tensor functor $F_{K}: \operatorname{Rep}(H) \rightarrow{ }_{K} \mathcal{M}_{K}$, we obtain a $\operatorname{Rep}(H)$ symmetric TQFT canonically from the data of a $\operatorname{Rep}(H)$-module category ${ }_{K} \mathcal{M}$. This suggests that the TQFT obtained in this way is a $\operatorname{Rep}(H)$ symmetric TQFT labeled by a module category ${ }_{K} \mathcal{M}$, or equivalently, this is a $\operatorname{Rep}(H)$ symmetric TQFT whose category of boundary conditions is given by ${ }_{K} \mathcal{M}$. In the next section, we will see that this is the case by showing that the action of the $\operatorname{Rep}(H)$ symmetry on the boundary conditions of this TQFT is described by the $\operatorname{Rep}(H)$-module action on ${ }_{K} \mathcal{M}$.

\section{State sum TQFTs and commuting projector Hamiltonians}

The canonical ${ }_{K} \mathcal{M}_{K}$ symmetric $\mathrm{TQFT}_{K} \mathcal{M}$ is obtained by state sum construction [60] whose input datum is a semisimple algebra $K$. The ${ }_{K} \mathcal{M}_{K}$ symmetry of this TQFT was first discussed in [65]. This symmetry can also be understood from a viewpoint of generalized gauging $[8-13,37]$. In this section, we show that this state sum TQFT actually has $\operatorname{Rep}(H)$ symmetry when the input algebra $K$ is a left $H$-comodule algebra. Specifically, this TQFT is regarded as the pullback of a ${ }_{K} \mathcal{M}_{K}$ symmetric TQFT ${ }_{K} \mathcal{M}$ by a tensor functor $F_{K}: \operatorname{Rep}(H) \rightarrow{ }_{K} \mathcal{M}_{K}$. We also construct $\operatorname{Rep}(H)$ symmetric commuting projector Hamiltonians whose ground states are described by the above state sum TQFTs. These commuting projector Hamiltonians realize all the gapped phases with non-anomalous fusion category symmetries.

\subsection{State sum TQFTs with defects}

We begin with reviewing state sum TQFTs with defects following [65]. We slightly modify the description of topological junctions in [65] so that it fits into the context of TQFTs with fusion category symmetries discussed in section 3 .

Let $\Sigma$ be a two-dimensional surface with in-boundary $\partial_{\text {in }} \Sigma$ and out-boundary $\partial_{\text {out }} \Sigma$. The surface $\Sigma$ is decorated by a network of topological defects that are labeled by objects of the category ${ }_{K} \mathcal{M}_{K}$. We assume that the junctions of these topological defects are trivalent and labeled by morphisms of ${ }_{K} \mathcal{M}_{K}$. We further assume, as in section 3.1, that

\footnotetext{
${ }^{11}$ More generally, we can construct all the TQFTs with arbitrary fusion category symmetries including anomalous ones just by replacing a Hopf algebra $H$ with a (semisimple pseudo-unitary connected) weak Hopf algebra in the following discussion, see also section 4.6 .
} 
the topological defects intersecting the in-boundary (out-boundary) are oriented so that they go into (out of) $\Sigma$

To assign a linear map to $\Sigma$, we first give a triangulation $T(\Sigma)$ of $\Sigma$ such that every face $p$ contains at most one trivalent junction and every edge $e$ intersects at most one topological defect. The possible configurations of topological defects on a face $p$ are as follows:

(i)

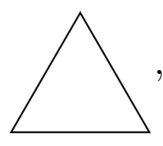

(ii)

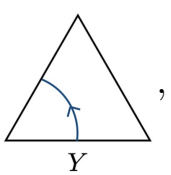

(iii)

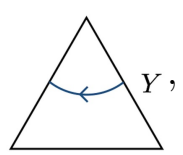

(iv)

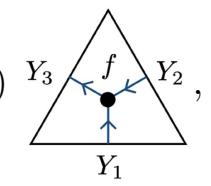

(v)

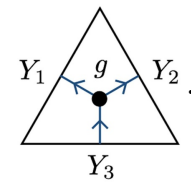

Here, topological defects are labeled by $K-K$ bimodules $Y, Y_{1}, Y_{2}, Y_{3} \in{ }_{K} \mathcal{M}_{K}$, and trivalent junctions are labeled by bimodule maps $f \in \operatorname{Hom}_{K K}\left(Y_{1} \otimes_{K} Y_{2}, Y_{3}\right), g \in \operatorname{Hom}_{K K}\left(Y_{3}, Y_{1} \otimes_{K}\right.$ $Y_{2}$ ). We note that all of the above configurations are obtained from configuration (iv) by choosing some of the topological defects as trivial defects or replacing some of the topological defects with their duals. Nevertheless, we distinguish these configurations for convenience.

For the triangulated surface $T(\Sigma)$, we define a linear map $Z_{T}(\Sigma)$ as [65]

$$
Z_{T}(\Sigma): Z_{T}\left(\partial_{\text {in }} \Sigma\right) \stackrel{\text { id } \otimes P(\Sigma)}{\longrightarrow} Z_{T}\left(\partial_{\text {in }} \Sigma\right) \otimes Q(\Sigma) \otimes Z_{T}\left(\partial_{\text {out }} \Sigma\right) \stackrel{E(\Sigma) \otimes \text { id }}{\longrightarrow} Z_{T}\left(\partial_{\text {out }} \Sigma\right) .
$$

The constituents of this linear map are described below.

The vector spaces $Z_{T}\left(\partial_{\text {in }} \Sigma\right)$ and $Z_{T}\left(\partial_{\text {out }} \Sigma\right)$. The vector space $Z_{T}\left(\partial_{a} \Sigma\right)$ for $a=$ in, out is defined as the tensor product of vector spaces $R_{e}$ assigned to edges $e \in \partial_{a} \Sigma$, namely

$$
Z_{T}\left(\partial_{a} \Sigma\right):=\bigotimes_{e \in \partial_{a} \Sigma} R_{e}
$$

where the vector spaces $R_{e}$ are given as follows:

$$
R_{e}:= \begin{cases}K & \text { when } e \text { does not intersect a topological defect } \\ Y & \text { when } e \text { intersects a topological defect } Y \in{ }_{K} \mathcal{M}_{K}\end{cases}
$$

We recall that the orientation of a topological defect $Y$ on a boundary edge $e \in \partial_{a} \Sigma$ is uniquely determined by assumption.

The vector space $Q(\Sigma)$. Similarly, we define the vector space $Q(\Sigma)$ as the tensor product of the vector spaces $Q_{(p, e)}$ assigned to flags $(p, e) \in \Sigma$ except for those whose edge $e$ is contained in the in-boundary $\partial_{\text {in }} \Sigma$ :

$$
Q(\Sigma):=\bigotimes_{(p, e) \in \Sigma \text { s.t. } e \notin \partial_{\mathrm{in}} \Sigma} Q_{(p . e)} .
$$

Here, a flag $(p, e)$ is a pair of a face $p$ and an edge $e$ on the boundary of $p$. The vector space $Q_{(p, e)}$ depends on both the label and the orientation of a topological defect 
that intersects the edge $e$. Concretely, we define

$$
Q_{(p, e)}:= \begin{cases}K & \text { when } e \text { does not intersect a topological defect } \\ Y & \text { when a topological defect } Y \text { goes into } p \text { across } e \\ Y^{*} & \text { when a topological defect } Y \text { goes out of } p \text { across } e\end{cases}
$$

The linear map $\boldsymbol{P}(\boldsymbol{\Sigma})$. The linear map $P(\Sigma): \mathbb{C} \rightarrow Q(\Sigma) \otimes Z_{T}\left(\partial_{\text {out }} \Sigma\right)$ is also defined in the form of the tensor product

$$
P(\Sigma):=\bigotimes_{e \in \Sigma \backslash \partial_{\mathrm{in}} \Sigma} P_{e}
$$

where the tensor product is taken over all edges $e$ of $\Sigma$ except for those on the in-boundary. The linear map $P_{e}$ for each edge $e \in \Sigma \backslash \partial_{\text {in }} \Sigma$ is given by

$$
P_{e}:= \begin{cases}\Delta_{K} \circ \eta_{K} & \text { when } e \text { does not intersect a topological defect } \\ \operatorname{coev}_{Y} & \text { when } e \text { intersects a topological defect } Y,\end{cases}
$$

where $\Delta_{K}: K \rightarrow K \otimes K$ and $\eta_{K}: \mathbb{C} \rightarrow K$ are the comultiplication and the unit of the Frobenius algebra $K$, see section 2.1. The coevaluation map $\operatorname{coev}_{Y}: \mathbb{C} \rightarrow Y \otimes Y^{*}$ is given by the usual embedding analogous to eq. (2.13).

The linear map $\boldsymbol{E}(\boldsymbol{\Sigma})$. Finally, the linear map $E(\Sigma): Z_{T}\left(\partial_{\mathrm{in}} \Sigma\right) \otimes Q(\Sigma) \rightarrow \mathbb{C}$ is again given by the tensor product

$$
E(\Sigma):=\bigotimes_{p \in \Sigma} E_{p}
$$

where the linear map $E_{p}$ for each face $p \in \Sigma$ depends on a configuration of topological defects on $p$. We have five different configurations (i)-(v) as shown in eq. (4.1), and define the linear map $E_{p}$ for each of them as follows:

$$
E_{p}:= \begin{cases}(\mathrm{i}) & \epsilon_{K} \circ m_{K} \circ\left(m_{K} \otimes \mathrm{id}_{K}\right): K \otimes K \otimes K \rightarrow \mathbb{C}, \\ (\mathrm{ii}) & \mathrm{ev}_{Y} \circ\left(\mathrm{id}_{Y^{*}} \otimes \rho_{Y}^{R}\right): Y^{*} \otimes Y \otimes K \rightarrow \mathbb{C}, \\ (\mathrm{iii}) & \mathrm{ev}_{Y} \circ\left(\mathrm{id}_{Y^{*}} \otimes \rho_{Y}^{L}\right): Y^{*} \otimes K \otimes Y \rightarrow \mathbb{C}, \\ (\mathrm{iv}) & \mathrm{ev}_{Y_{3}} \circ\left(\operatorname{id}_{Y_{3}^{*}} \otimes\left(f \circ \pi_{Y_{1}, Y_{2}}\right)\right): Y_{3}^{*} \otimes Y_{1} \otimes Y_{2} \rightarrow \mathbb{C}, \\ (\mathrm{v}) & \mathrm{ev}_{Y_{1} \otimes Y_{2}} \circ\left(\operatorname{id}_{\left(Y_{1} \otimes Y_{2}\right)^{*}} \otimes\left(\iota_{Y_{1}, Y_{2}} \circ g\right)\right):\left(Y_{1} \otimes Y_{2}\right)^{*} \otimes Y_{3} \rightarrow \mathbb{C} .\end{cases}
$$

Here, $\rho_{Y}^{L}: K \otimes Y \rightarrow Y$ and $\rho_{Y}^{R}: Y \otimes K \rightarrow Y$ denote the left and right $K$-module actions on $Y$ respectively, and $\pi_{Y_{1}, Y_{2}}$ and $\iota_{Y_{1}, Y_{2}}$ are the splitting maps defined in section 2.1. The linear maps $f \in \operatorname{Hom}_{K K}\left(Y_{1} \otimes_{K} Y_{2}, Y_{3}\right)$ and $g \in \operatorname{Hom}_{K} K\left(Y_{3}, Y_{1} \otimes_{K} Y_{2}\right)$ are morphisms in the category of $K-K$ bimodules. As we mentioned before, the linear maps for (i)-(iii) and (v) are obtained from that for (iv) with an appropriate choice of $Y_{1}, Y_{2}, Y_{3}$, and $f$. 
Combining the above definitions (4.3)-(4.10), we obtain the linear map $Z_{T}(\Sigma)$ : $Z_{T}\left(\partial_{\text {in }} \Sigma\right) \rightarrow Z_{T}\left(\partial_{\text {out }} \Sigma\right)$ via eq. (4.2). However, $Z_{T}(\Sigma)$ is not yet an appropriate transition amplitude of a TQFT because the linear map $Z_{T}\left(\partial_{\text {in }} \Sigma \times[0,1]\right)$ assigned to a cylinder $\partial_{\text {in }} \Sigma \times[0,1]$ is not the identity map. In particular, the linear map $Z_{T}\left(\partial_{\text {in }} \Sigma \times[0,1]\right)$ is an idempotent on $Z_{T}\left(\partial_{\text {in }} \Sigma\right)$, whose image will be denoted by $Z\left(\partial_{\text {in }} \Sigma\right)$. It turns out that $Z\left(\partial_{\text {in }} \Sigma\right)$ is mapped to $Z\left(\partial_{\text {out }} \Sigma\right)$ by $Z_{T}(\Sigma)$. Hence, we obtain a linear map $Z(\Sigma): Z\left(\partial_{\text {in }} \Sigma\right) \rightarrow Z\left(\partial_{\text {out }} \Sigma\right)$ by restricting the domain of the linear map (4.2) to $Z\left(\partial_{\text {in }} \Sigma\right)$. We note that the linear map assigned to a cylinder is now the identity map. It is shown in [65] that the assignment of the vector spaces $Z\left(\partial_{\text {in/out }} \Sigma\right)$ and the linear map $Z(\Sigma)$ gives a TQFT with defects. ${ }^{12}$ Based on the above definition, we find that the two possible ways to resolve a quadrivalent junction into two trivalent junctions are related by the associator $\alpha_{Y_{1}, Y_{2}, Y_{3}}$ defined by eq. (2.16) as follows:

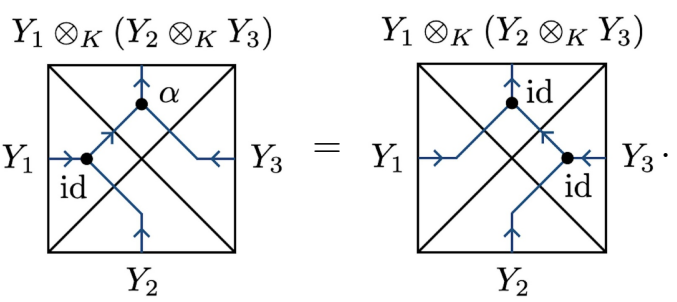

The square in the above equation represents a local patch of an arbitrary triangulated surface. This equation (4.11) implies that the symmetry of the state sum TQFT is precisely described by ${ }_{K} \mathcal{M}_{K}$.

To argue that the state sum TQFT obtained above is the canonical ${ }_{K} \mathcal{M}_{K}$ symmetric TQFT $_{K} \mathcal{M}$, we first notice that the state sum construction can be viewed as a generalized gauging of the trivial TQFT [37]. Here, the generalized gauging of a TQFT $Q$ with fusion category symmetry $\mathcal{C}$ is the procedure to condense a $\Delta$-separable symmetric Frobenius algebra object $A \in \mathcal{C}$ on a two-dimensional surface. This procedure gives rise to a new TQFT $Q / A$ whose symmetry is given by the category ${ }_{A} \mathcal{C}_{A}$ of $A$ - $A$ bimodules in $\mathcal{C}[12,13]$. To examine the relation between $Q$ and $Q / A$ in more detail, we consider the categories of boundary conditions of these TQFTs. Let $\mathcal{B}$ be the category of boundary conditions of the original TQFT $Q$. We note that $\mathcal{B}$ is the category of right $B$-modules in $\mathcal{C}$ for some $\Delta$-separable symmetric Frobenius algebra object $B \in \mathcal{C}$ because $\mathcal{B}$ is a left $\mathcal{C}$-module category $[72,73]$. Then, the category of boundary conditions of the gauged TQFT $Q / A$ should be the category of left $A$-modules in $\mathcal{B}$ [19], which is a left ${ }_{A} \mathcal{C}_{A}$-module category. This is because the algebra object $A$ is condensed in the gauged theory and hence a boundary condition in $\mathcal{B}$ survives after gauging only when it is a left $A$-module. ${ }^{13}$ For example, gauging the algebra object $H^{*}$ in a $\operatorname{Rep}(H)$ symmetric TQFT ${ }_{K} \mathcal{M}$ would result in a $\operatorname{Rep}\left(H^{*}\right)$ symmetric TQFT $\left(H^{*} \mathcal{M}\right)_{K^{\text {op }}} \cong \operatorname{Stab}_{K^{\text {op }}(V)} \mathcal{M}$ due to Theorem 3.10. of [58],14 where $K$ is an $H$-simple left $H$-comodule algebra, $V$ is a left $K^{\mathrm{op}}$-module, and $\operatorname{Stab}_{K^{\mathrm{op}}}(V)$

\footnotetext{
${ }^{12}$ The proof of the topological invariance in [65] can still be applied even though the description of topological junctions is slightly changed.

${ }^{13}$ The reason why we use left $A$-modules instead of right $A$-modules is that the category $\mathcal{B}$ of boundary conditions is supposed to be a left module category over $\mathcal{C}$.

${ }^{14}$ This generalized gauging is relevant for relating the state sum models to the anyon chain models as we mentioned in section 1 .
} 


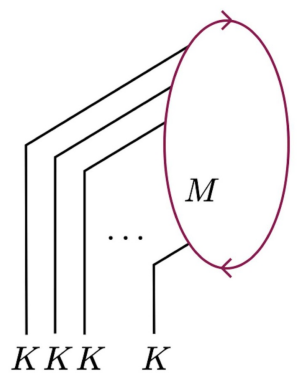

Figure 3. The boundary state (4.13) is represented by the above string diagram where the circle labeled by $M$ corresponds to taking the trace on $M$. This string diagram is invariant under the composition of the cylinder amplitude $Z_{T}\left(S^{1} \times[0,1]\right)$ at the bottom.

is the Yan-Zhu stabilizer of $K^{\mathrm{op}}$ with respect to $V$ [76]. The $\operatorname{Rep}\left(H^{*}\right)$-module category $\operatorname{Stab}_{K^{\mathrm{op}}(V)} \mathcal{M}$ does not depend on a choice of $V$ [58]. In the case of the state sum TQFT with the input $K$, the condensed algebra object is $K \in$ Vec and the category of boundary conditions of the original TQFT is Vec. Therefore, the category of boundary conditions of the state sum TQFT would be the category ${ }_{K} \mathcal{M}$ of left $K$-modules.

We can also see this more explicitly by computing the action of the ${ }_{K} \mathcal{M}_{K}$ symmetry on the boundary states of the state sum TQFT. For this purpose, we first notice that a boundary of the state sum TQFT is equivalent to an interface between the state sum TQFT and the trivial TQFT. Since the trivial TQFT is a state sum TQFT with the trivial input $\mathbb{C}$, interfaces are described by $K-\mathbb{C}$ bimodules, or equivalently, left $K$-modules. The wave function of the boundary state $\langle M|$ corresponding to the boundary condition $M \in{ }_{K} \mathcal{M}$ is the linear map assigned to a triangulated disk

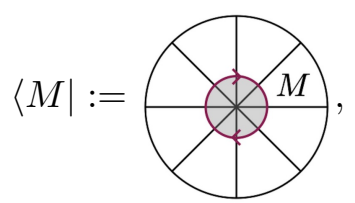

where the outer circle is an in-boundary and the inner circle labeled by $M$ is the interface between the trivial TQFT (shaded region) and the state sum TQFT with the input $K$ (unshaded region). We can compute the linear map assigned to the above disk by using a left $K$-module $M$ instead of a $K$ - $K$ bimodule $Y$ in eqs. (4.4), (4.6), (4.8), and (4.10) [65], see also appendix A for more details. Specifically, we can express the wave function $\langle M|$ in the form of a matrix product state (MPS) as [75, 77]

$$
\langle M|=\sum_{i_{1}, \cdots, i_{N}} \operatorname{tr}\left[T_{M}\left(e_{i_{1}}\right) \cdots T_{M}\left(e_{i_{N}}\right)\right]\left\langle e_{i_{1}}, \cdots, e_{i_{N}}\right|,
$$

where $\left\{e_{i}\right\}$ is a basis of $K, N$ is the number of edges on the boundary, and $T_{M}: K \rightarrow$ $\operatorname{End}(M)$ is the $K$-module action on $M$. In the string diagram notation, this MPS can be represented as shown in figure 3. We notice that the MPS (4.13) satisfies the additive property

$$
\left\langle M_{1} \oplus M_{2}\right|=\left\langle M_{1}\right|+\left\langle M_{2}\right|,
$$

due to which it suffices to consider simple modules $M_{j} \in{ }_{K} \mathcal{M}$. 
A topological defect $Y \in{ }_{K} \mathcal{M}_{K}$ acts on a boundary state $\left\langle M_{j}\right|$ by winding around the spatial circle. We denote the wave function of the resulting state by $\left\langle Y \cdot M_{j}\right|$. By giving a specific triangulation of a disk as follows, we can compute the action of $Y$ on the boundary state $\left\langle M_{j}\right|$ as

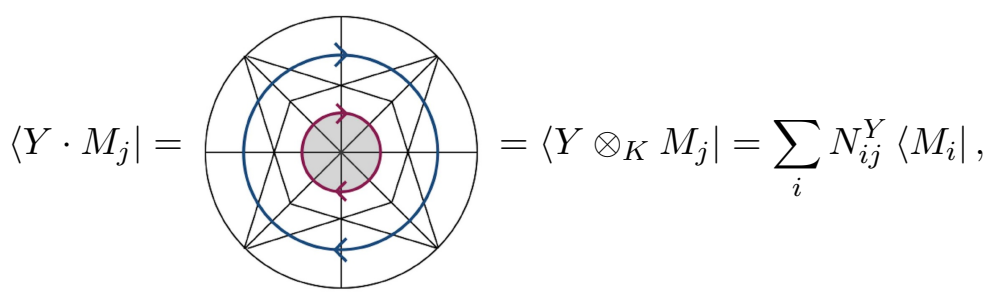

where the blue circle and the purple circle represent a topological defect $Y \in{ }_{K} \mathcal{M}_{K}$ and a boundary condition $M_{j} \in{ }_{K} \mathcal{M}$ respectively, and $N_{i j}^{Y}$ is a non-negative integer that appears in the direct sum decomposition of $Y \otimes_{K} M_{j} \cong \bigoplus_{i} N_{i j}^{Y} M_{i}$. We note that the boundary states form a non-negative integer matrix representation (NIM rep) of the fusion ring of ${ }_{K} \mathcal{M}_{K}$. Equation (4.15) implies that the action of the ${ }_{K} \mathcal{M}_{K}$ symmetry on boundary conditions is described by a module category ${ }_{K} \mathcal{M}$. The module associativity constraint $(2.17)$ is also captured in the same way as eq. (4.11). Thus, the category of boundary conditions is given by the ${ }_{K} \mathcal{M}_{K}$-module category ${ }_{K} \mathcal{M}$, which indicates that the state sum TQFT with the input $K$ is a ${ }_{K} \mathcal{M}_{K}$ symmetric $\mathrm{TQFT}_{K} \mathcal{M}$.

\subsection{Pullback of state sum TQFTs}

When $K$ is a left $H$-comodule algebra, the ${ }_{K} \mathcal{M}_{K}$ symmetric TQFT ${ }_{K} \mathcal{M}$ can be pulled back to a $\operatorname{Rep}(H)$ symmetric TQFT by a tensor functor $F_{K}: \operatorname{Rep}(H) \rightarrow{ }_{K} \mathcal{M}_{K}$. Accordingly, the symmetry of the state sum TQFT with the input $K$ can be regarded as $\operatorname{Rep}(H)$. Specifically, when a two-dimensional surface $\Sigma$ is decorated by a topological defect network associated with the $\operatorname{Rep}(H)$ symmetry, the assignment of the vector spaces (4.4), (4.6) and linear maps (4.8), (4.10) are modified as follows:

$$
\begin{aligned}
R_{e} & := \begin{cases}K & \text { when } e \text { does not intersect a topological defect, } \\
F_{K}(V) & \text { when } e \text { intersects a topological defect } V \in \operatorname{Rep}(H) .\end{cases} \\
Q_{(p, e)} & := \begin{cases}K & \text { when } e \text { does not intersect a topological defect, } \\
F_{K}(V) & \text { when a topological defect } V \text { goes into } p \text { across } e, \\
F_{K}(V)^{*} & \text { when a topological defect } V \text { goes out of } p \text { across } e .\end{cases} \\
P_{e} & := \begin{cases}\Delta_{K} \circ \eta_{K} & \text { when } e \text { does not intersect a topological defect, } \\
\operatorname{coev}_{F_{K}(V)} & \text { when } e \text { intersects a topological defect } V .\end{cases}
\end{aligned}
$$




$$
E_{p}:= \begin{cases}(\text { i }) & \epsilon_{K} \circ m_{K} \circ\left(m_{K} \otimes \operatorname{id}_{K}\right), \\ \text { (ii) } & \operatorname{ev}_{F_{K}(V)} \circ\left(\operatorname{id}_{F_{K}(V)^{*}} \otimes \rho_{F_{K}(V)}^{R}\right), \\ (\text { iii }) & \operatorname{ev}_{F_{K}(V)} \circ\left(\operatorname{id}_{F_{K}(V)^{*}} \otimes \rho_{F_{K}(V)}^{L}\right), \\ \text { (iv) } & \operatorname{ev}_{F_{K}\left(V_{3}\right)} \circ\left(\operatorname{id}_{F_{K}\left(V_{3}\right)^{*}} \otimes\left(F_{K}(f) \circ J_{V_{1}, V_{2}} \circ \pi_{F_{K}\left(V_{1}\right), F_{K}\left(V_{2}\right)}\right)\right), \\ (\mathrm{v}) & \operatorname{ev}_{F_{K}\left(V_{1}\right) \otimes F_{K}\left(V_{2}\right)} \circ\left(\operatorname{id}_{\left(F_{K}\left(V_{1}\right) \otimes F_{K}\left(V_{2}\right)\right)^{*}} \otimes\left(\iota_{F_{K}\left(V_{1}\right), F_{K}\left(V_{2}\right)} \circ J_{V_{1}, V_{2}}^{-1} \circ F_{K}(g)\right)\right) .\end{cases}
$$

(i)-(v) in eq. (4.19) refer to the configurations (4.1) of topological defects on a face $p$, where the topological defects and junctions of ${ }_{K} \mathcal{M}_{K}$ symmetry are replaced with those of $\operatorname{Rep}(H)$ symmetry, i.e. $V, V_{1}, V_{2}, V_{3} \in \operatorname{Rep}(H), f \in \operatorname{Hom}_{\operatorname{Rep}(H)}\left(V_{1} \otimes V_{2}, V_{3}\right)$, and $g \in$ $\operatorname{Hom}_{R e p(H)}\left(V_{3}, V_{1} \otimes V_{2}\right)$. The linear map $J_{V_{1}, V_{2}}: F_{K}\left(V_{1}\right) \otimes_{K} F_{K}\left(V_{2}\right) \rightarrow F_{K}\left(V_{1} \otimes V_{2}\right)$ is a natural isomorphism associated with the tensor functor $F_{K}: \operatorname{Rep}(H) \rightarrow{ }_{K} \mathcal{M}_{K}$. Here, we again point out that (i)-(iii) and (v) are obtained from (iv) by choosing topological defects and junctions appropriately. By substituting the above definitions to eq. (4.2), we assign a linear map $Z_{T}(\Sigma)$ to a triangulated surface $\Sigma$ decorated by defects of the $\operatorname{Rep}(H)$ symmetry. Then, as in the previous subsection, we obtain a TQFT with $\operatorname{Rep}(H)$ symmetry by restricting the domain of the linear map $Z_{T}(\Sigma)$ to the image of the cylinder amplitude $Z_{T}\left(\partial_{\text {in }} \Sigma \times[0,1]\right)$. The topological invariance of this $\operatorname{Rep}(H)$ symmetric TQFT readily follows from the topological invariance of the ${ }_{K} \mathcal{M}_{K}$ symmetric state sum TQFT defined in the previous subsection. It is also straightforward to check that the difference between two possible resolutions of a quadrivalent junction into two trivalent junctions is described by the associator of $\operatorname{Rep}(H)$.

We can compute the action of the $\operatorname{Rep}(H)$ symmetry on the boundary states as follows. We first recall that the action of the ${ }_{K} \mathcal{M}_{K}$ symmetry on the boundary states is represented by the ${ }_{K} \mathcal{M}_{K}$-module action on ${ }_{K} \mathcal{M}$ as eq. (4.15). This ${ }_{K} \mathcal{M}_{K}$ action induces a $\operatorname{Rep}(H)$ action on the boundary states because topological defects associated with the $\operatorname{Rep}(H)$ symmetry are mapped to ${ }_{K} \mathcal{M}_{K}$ symmetry defects by a tensor functor $F_{K}: \operatorname{Rep}(H) \rightarrow$ ${ }_{K} \mathcal{M}_{K}$. Specifically, a topological defect $V \in \operatorname{Rep}(H)$ acts on a boundary state $\langle M|$ as

$$
\langle V \cdot M|=\left\langle F_{K}(V) \otimes_{K} M\right|=\langle V \bar{\otimes} M|,
$$

where $\bar{\otimes}$ represents the $\operatorname{Rep}(H)$-module action on ${ }_{K} \mathcal{M}$, see section 2.3. Combined with the additive property (4.14), this equation implies that the boundary states form a NIM rep of the fusion ring of $\operatorname{Rep}(H)$. Furthermore, if we extend the definition (4.19) in the presence of boundaries as we describe in appendix A, we find that the two possible resolutions of a junction on the boundary are related to each other by the module associativity constraint. Therefore, the category of boundary conditions of this TQFT is given by a $\operatorname{Rep}(H)$-module category ${ }_{K} \mathcal{M}$. Since every semisimple indecomposable module category over $\operatorname{Rep}(H)$ is equivalent to the category ${ }_{K} \mathcal{M}$ of left modules over an $H$-simple left $H$-comodule algebra $K$, we conclude that any semisimple indecomposable TQFTs with $\operatorname{Rep}(H)$ symmetry are obtained via the above state sum construction. ${ }^{15}$ Here, we recall that an $H$-simple left $H$-comodule algebra is semisimple [61] and hence can be used as an input of the state sum construction.

\footnotetext{
${ }^{15}$ The state sum TQFTs with ordinary finite group symmetry $G$ are originally discussed in $[75,77,78]$. We can reproduce these TQFTs as special cases where the Hopf algebra $H$ is a dual group algebra $\mathbb{C}[G]^{*}$.
} 


\subsection{Commuting projector Hamiltonians}

In this subsection, we write down $\operatorname{Rep}(H)$ symmetric commuting projector Hamiltonians whose ground states are described by the $\operatorname{Rep}(H)$ symmetric $\mathrm{TQFTs}_{K} \mathcal{M}$. For concreteness, we choose $K=A^{\mathrm{op}} \# H^{\text {cop }}$ where $A$ is an $H$-simple left $H$-module algebra. This is always possible because every semisimple indecomposable module category over $\operatorname{Rep}(H)$ is equivalent to $A^{\mathrm{op}} \# H^{\mathrm{cop}} \mathcal{M}$ as we discussed in section 2.3. We first define the Hilbert space on a circular lattice $T\left(S^{1}\right)$, which is a triangulation of a circle $S^{1}$. The Hilbert space $\mathcal{H}$ on the lattice $T\left(S^{1}\right)$ is the tensor product of local Hilbert spaces $K_{i}:=K$ on edges $i=1,2, \cdots, N$, i.e. $\mathcal{H}:=\bigotimes_{i} K_{i}$. We define a commuting projector Hamiltonian $H$ acting on this Hilbert space $\mathcal{H}$ as $[62,63]^{16}$

$$
H:=\sum_{i}\left(1-h_{i, i+1}\right), \quad h_{i, i+1}:=\Delta_{K} \circ m_{K}: K_{i} \otimes K_{i+1} \rightarrow K_{i} \otimes K_{i+1},
$$

where the comultiplication $\Delta_{K}$ for the Frobenius algebra structure on $K$ is given by eq. (2.12). The fact that $K$ is a $\Delta$-separable symmetric Frobenius algebra (2.14) guarantees that the linear map $h_{i, i+1}$ becomes a local commuting projector, i.e. $h_{i, i+1} h_{j, j+1}=$ $h_{j, j+1} h_{i, i+1}$ and $h_{i, i+1}^{2}=h_{i, i+1}$. The local commuting projector $h_{i, i+1}$ can also be written in terms of a string diagram as

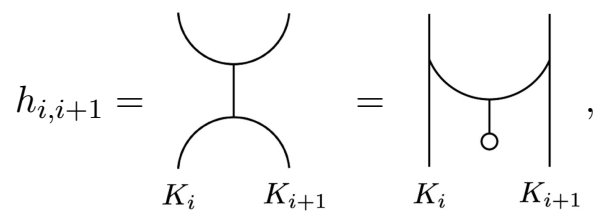

where we used the Frobenius relation (2.11). The projector $\Pi$ to the subspace of $\mathcal{H}$ spanned by the ground states of the Hamiltonian (4.21) is given by the composition of the local commuting projectors $h_{i, i+1}$ for all edges $i=1,2, \cdots, N$. This projector $\Pi: \mathcal{H} \rightarrow \mathcal{H}$ can be represented by the following string diagram:

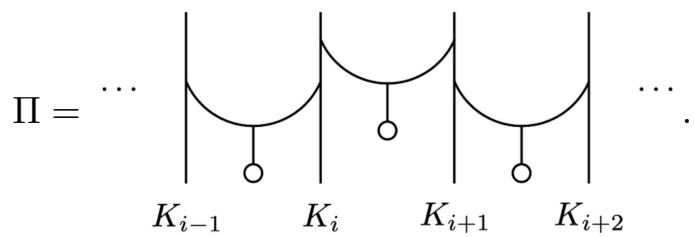

This coincides with the string diagram representation of the linear map $Z_{T}\left(S^{1} \times[0,1]\right)$ assigned to a triangulated cylinder $S^{1} \times[0,1]$. Therefore, the ground states of the commuting projector Hamiltonian (4.21) agree with the vacua of the state sum TQFT whose input algebra is $K$.

This is because an ordinary finite group symmetry $G$ is described by $\operatorname{Rep}\left(\mathbb{C}[G]^{*}\right)$ as a fusion category symmetry. We note that a left $\mathbb{C}[G]^{*}$-comodule algebra $K$ is a $G$-equivariant algebra, which agrees with the input algebra used in $[75,77,78]$.

${ }^{16}$ We can also define the Hilbert space and the Hamiltonian of a twisted sector analogously. Specifically, when a topological defect $V \in \operatorname{Rep}(H)$ intersects an edge $i$, the local Hilbert space on $i$ is given by $F_{K}(V)=V \otimes K$ according to eq. (4.16). Namely, we attach a vector space $V$ to a topological defect $V \in \operatorname{Rep}(H)$. This generalizes the twisted sectors of finite gauge theories discussed in [79]. 
We can define the action of the $\operatorname{Rep}(H)$ symmetry on the lattice Hilbert space $\mathcal{H}$ via the $H$-comodule structure on $K$. Concretely, the adjoint of the action $U_{V}: \mathcal{H} \rightarrow \mathcal{H}$ associated with a topological defect $V \in \operatorname{Rep}(H)$ is given by the following string diagram

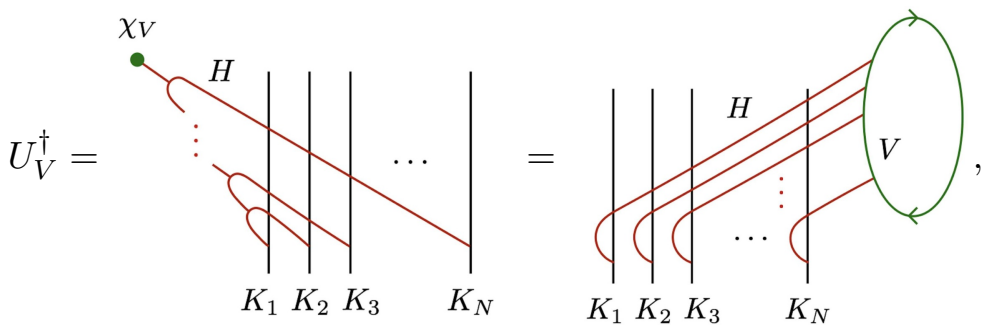

where $\chi_{V} \in H^{*}$ is the character of the representation $V \in \operatorname{Rep}(H)$, which is defined as the trace of the left $H$-module action on $V .{ }^{17}$ The above $\operatorname{Rep}(H)$ action obeys the fusion rule of $\operatorname{Rep}(H)$, i.e. $U_{V_{i}} U_{V_{j}}=\sum_{k} N_{i j}^{k} U_{V_{k}}$ for irreducible representations $V_{i}, V_{j} \in \operatorname{Rep}(H)$, where $N_{i j}^{k}$ is a fusion coefficient $V_{i} \otimes V_{j} \cong \bigoplus_{k} N_{i j}^{k} V_{k}$. The cyclic symmetry of the character guarantees that the action (4.24) is well-defined on a periodic lattice $T\left(S^{1}\right)$. Moreover, this action is faithful since the left $H$-comodule action on $K=A^{\mathrm{op}} \# H^{\mathrm{cop}}$ is inner-faithful. ${ }^{18}$

Let us now show the commutativity of the $\operatorname{Rep}(H)$ action (4.24) and the commuting projector Hamiltonian (4.21). It suffices to check that the $\operatorname{Rep}(H)$ action commutes with each local commuting projector $h_{i, i+1}$. Namely, we need to check

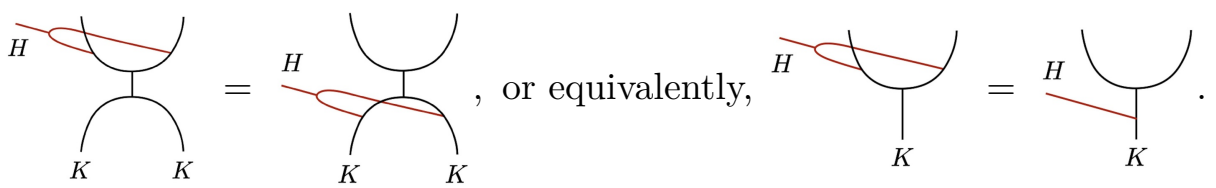

The first equality follows from the second equality because $K$ is a left $H$-comodule algebra. Conversely, we can derive the second equality from the first equality by composing a unit at the bottom of the diagram.

To show eq. (4.25), we first notice that the counit $\epsilon$ given by eq. (2.12) satisfies

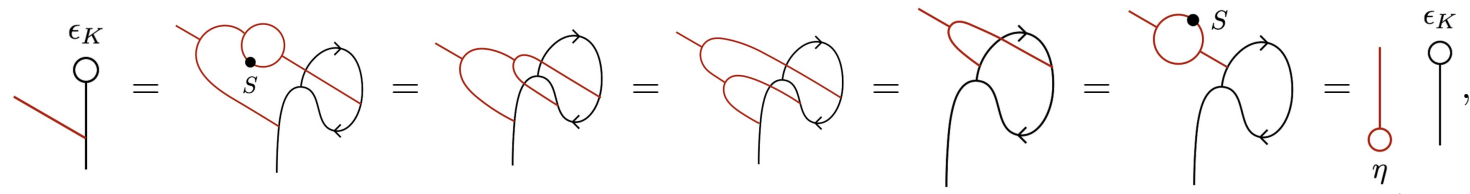

where we used the left $H$-comodule action on $K^{*}$ defined in a similar way to eq. (2.32). We note that the above equation relies on the fact that the antipode $S$ of a semisimple Hopf algebra $H$ squares to the identity. Equation (4.26) in turn implies that the isomorphism

\footnotetext{
${ }^{17}$ We note that the $\operatorname{Rep}(H)$ action $(4.24)$ does not involve the algebra structure on $K$, which means that we can define the $\operatorname{Rep}(H)$ action on the lattice as long as the local Hilbert space is a left $H$-comodule.

${ }^{18}$ Another choice of $K$ is also possible as long as the $\operatorname{Rep}(H)$ symmetry acts faithfully on the lattice Hilbert space.
} 
$\Phi: K \rightarrow K^{*}$ defined in eq. (2.12) is an $H$-comodule map because

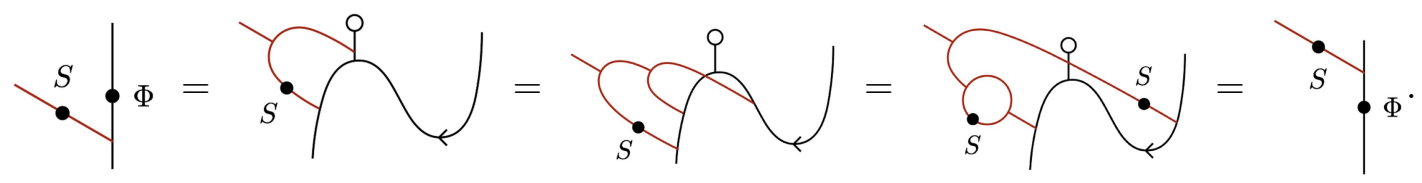

This indicates that $\Phi^{-1}: K^{*} \rightarrow K$ is an $H$-comodule map as well. Therefore, we have

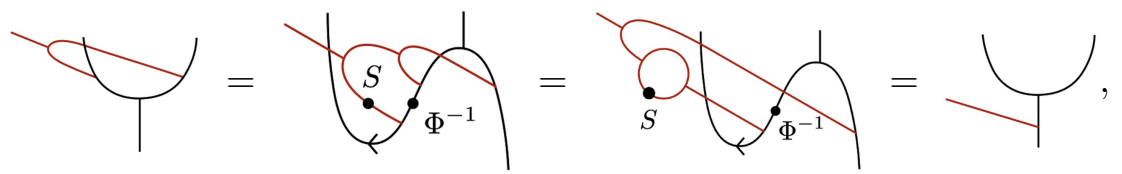

which shows eq. (4.25).

We can also compute the action (4.24) of the $\operatorname{Rep}(H)$ symmetry on the ground states of the Hamiltonian (4.21). To perform the computation, we recall that the ground states of (4.21) are in one-to-one correspondence with the vacua of the state sum TQFT, and hence can be written as the boundary states (4.13) [74]. The $\operatorname{Rep}(H)$ symmetry action $U_{V}$ on a boundary state $\langle M|$ is given by

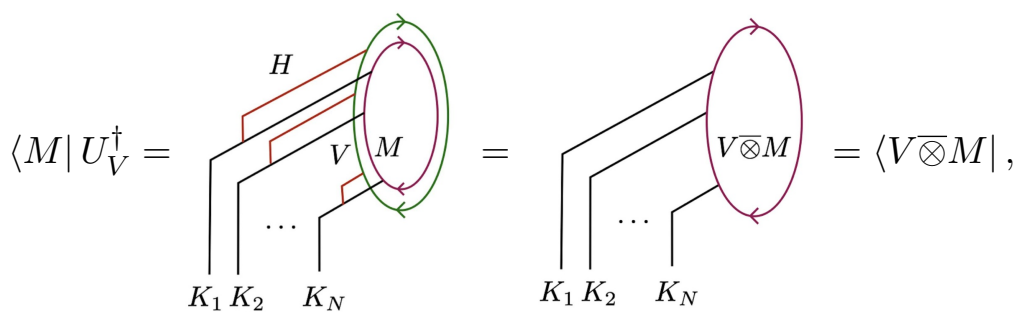

which coincides with the $\operatorname{Rep}(H)$ symmetry action (4.20) of the state sum TQFT. This implies that the commuting projector Hamiltonian (4.21) is a lattice realization of a $\operatorname{Rep}(H)$ symmetric TQFT ${ }_{K} \mathcal{M}$. In summary, every semisimple indecomposable TQFT ${ }_{K} \mathcal{M} \cong\left({ }_{H} \mathcal{M}\right)_{A}$ with $\operatorname{Rep}(H)$ symmetry can be realized by a commuting projector Hamiltonian (4.21) on the lattice where the input datum $K$ is an $H$-simple left $H$-comodule algebra $K=A^{\mathrm{op}} \# H^{\mathrm{cop}}$.

\subsection{Examples: gapped phases of finite gauge theories}

Let $G$ be a finite group and $\mathbb{C}[G]$ be a group algebra. Gapped phases of $G$ gauge theory are labeled by a pair $(H, \omega)[15]$ where $H$ is a subgroup of $G$ to which the gauge group $G$ is Higgsed down and $\omega \in H^{2}(H, \mathrm{U}(1))$ is a discrete torsion [80]. The symmetry of $G$ gauge theory is described by $\operatorname{Rep}(G):=\operatorname{Rep}(\mathbb{C}[G])$, which is generated by the Wilson lines. Therefore, we can realize these phases by the commuting projector Hamiltonians (4.21) where the input algebra $K$ is a left $\mathbb{C}[G]$-comodule algebra. Specifically, the input algebra $K$ for the gapped phase labeled by $(H, \omega)$ is given by $K=\left(\mathbb{C}[G] \otimes_{\mathbb{C}[H]} \operatorname{End}(U)\right)^{\mathrm{op}} \# \mathbb{C}[G]$, where $U$ is a projective representation of $H$ characterized by $\omega$ [58]. ${ }^{19}$ The action (4.24) of a representation $V \in \operatorname{Rep}(G)$ is expressed as

$$
U_{V}^{\dagger}\left|a_{1} \# g_{1}, a_{2} \# g_{2}, \cdots, a_{N} \# g_{N}\right\rangle=\chi_{V}\left(g_{1} g_{2} \cdots g_{N}\right)\left|a_{1} \# g_{1}, a_{2} \# g_{2}, \cdots, a_{N} \# g_{N}\right\rangle
$$

\footnotetext{
${ }^{19}$ We note that the group algebra $\mathbb{C}[G]$ is cocommutative, i.e. $\left.\mathbb{C}[G]\right]^{\text {cop }}=\mathbb{C}[G]$.
} 
for $a_{i} \in\left(\mathbb{C}[G] \otimes_{\mathbb{C}[H]} \operatorname{End}(U)\right)^{\text {op }}$ and $g_{i} \in G$. In the following, we will explicitly describe the actions of the commuting projector Hamiltonians (4.21) for gapped phases of $G$ gauge theory by choosing a specific basis of $K$. For simplicity, we will only consider two limiting cases where the gauge group $G$ is not Higgsed at all or completely Higgsed.

When $G$ is not Higgsed, the gapped phases of $G$ gauge theory are described by Dijkgraaf-Witten theories [81]. The input algebras $K$ for these phases are given by $K=\operatorname{End}(U)^{\mathrm{op}} \# \mathbb{C}[G] .{ }^{20} \quad$ We choose a basis of the algebra $K$ as $\left\{E_{i j} \# v_{g} \mid i, j=\right.$ $1,2, \cdots, \operatorname{dim} U, g \in G\}$, where $E_{i j}$ is a $\operatorname{dim} U \times \operatorname{dim} U$ matrix whose $(k, l)$ component is 1 when $(k, l)=(i, j)$ and otherwise 0 . If we denote the projective action of $G$ on $U$ by $Q: G \rightarrow \operatorname{End}(U)$, the multiplication $(2.30)$ on the algebra $K$ is written as

$$
\left(E_{i j} \# v_{g}\right) \cdot\left(E_{k l} \# v_{h}\right)=Q(g) E_{k l} Q(g)^{-1} E_{i j} \# v_{g h} .
$$

The Frobenius algebra structure on $K$ is characterized by a pairing

$$
\epsilon_{K}\left(\left(E_{i j} \# v_{g}\right) \cdot\left(E_{k l} \# v_{h}\right)\right)=|G| \operatorname{dim} U \delta_{g, h^{-1}}\left[Q(g) E_{k l} Q(g)^{-1}\right]_{j i},
$$

where the last term on the right-hand side represents the $(j, i)$ component of $Q(g) E_{k l} Q(g)^{-1}$. The above equation implies that $Q(g)^{-1} E_{j i} Q(g) \# v_{g^{-1}} /|G| \operatorname{dim} U$ is dual to $E_{i j} \# v_{g}$ with respect to the pairing $\epsilon_{K} \circ m_{K}$, and hence the comultiplication of the unit element $1_{K} \in K$ is given by

$$
\Delta_{K}\left(1_{K}\right)=\frac{1}{|G| \operatorname{dim} U} \sum_{i, j} \sum_{g} E_{i j} \# v_{g} \otimes Q(g)^{-1} E_{j i} Q(g) \# v_{g^{-1}} .
$$

Therefore, we can explicitly write down the action of the local commuting projector $h$ : $K \otimes K \rightarrow K \otimes K$ defined by eq. (4.22) as

$h\left(E_{i j} \# v_{g} \otimes E_{k l} \# v_{h}\right)=\frac{1}{|G| \operatorname{dim} U} \sum_{m} \sum_{f \in G} Q(g) E_{m l} Q(g)^{-1} E_{i j} \# v_{g f} \otimes Q(f)^{-1} E_{k m} Q(f) \# v_{f^{-1} h}$.

On the other hand, when $G$ is completely Higgsed, the input algebra $K$ is given by $K=\mathbb{C}[G]^{*} \# \mathbb{C}[G]$. We choose a basis of $K$ as $\left\{v^{g} \# v_{h} \mid g, h \in G\right\}$ where $v^{g} \in \mathbb{C}[G]^{*}$ denotes the dual basis of $v_{g} \in \mathbb{C}[G]$. The multiplication (2.30) on the algebra $K$ is written as

$$
\left(v^{g} \# v_{h}\right) \cdot\left(v^{k} \# v_{l}\right)=\delta_{g, h k} v^{g} \# v_{h l},
$$

where we defined a left $\mathbb{C}[G]$-module action on $\mathbb{C}[G]^{*}$ by the left translation $\rho\left(v_{g}\right) v^{h}:=$ $v^{g h}$. Since the dual of $v^{g} \# v_{h}$ with respect to the Frobenius pairing $\epsilon_{K} \circ m_{K}$ is given by $v^{h^{-1} g} \# v_{h^{-1}} /|G|$, we have

$$
\Delta_{K}\left(1_{K}\right)=\frac{1}{|G|} \sum_{g, h} v^{g} \# v_{h} \otimes v^{h^{-1} g} \# v_{h^{-1}} .
$$

Thus, the action of the local commuting projector $h$ is expressed as

$$
h\left(v^{g} \# v_{h} \otimes v^{k} \# v_{l}\right)=\frac{\delta_{g, h k}}{|G|} \sum_{m} v^{g} \# v_{h m} \otimes v^{m^{-1} k} \# v_{m^{-1} l} .
$$

\footnotetext{
${ }^{20}$ The input algebra of a Dijkgraaf-Witten theory is usually chosen as a twisted group algebra $\mathbb{C}[G]^{\omega}$. The algebras $\mathbb{C}[G]^{\omega}$ and $\operatorname{End}(U)^{\mathrm{op}} \# \mathbb{C}[G]$ give rise to the same TQFT because $\mathbb{C}[G]^{\omega} \mathcal{M}$ is equivalent to $\operatorname{End}(U)^{\mathrm{op}} \# \mathbb{C}[G] \mathcal{M}$ as a module category over $\operatorname{Rep}(G)[58]$.
} 


\subsection{Edge modes of SPT phases with fusion category symmetries}

SPT phases with fusion category symmetry $\mathcal{C}$ are uniquely gapped phases preserving the symmetry $\mathcal{C}$. Since anomalous fusion category symmetries do not admit SPT phases, it suffices to consider non-anomalous symmetries $\mathcal{C}=\operatorname{Rep}(H)$. SPT phases with $\operatorname{Rep}(H)$ symmetry are realized by the commuting projector Hamiltonians (4.21) when $K=A^{\text {op }} \# H^{\text {cop }}$ is a simple algebra. ${ }^{21}$ These Hamiltonians have degenerate ground states on an interval even though they have unique ground states on a circle. Specifically, it turns out that the ground states on an interval are given by the algebra $K[64,65]$. Since $K$ is simple, we can write $K \cong \operatorname{End}(M) \cong M^{*} \otimes M$ where $M$ is a simple left $K$-module, which is unique up to isomorphism. We can interpret $M^{*}$ and $M$ as the edge modes localized to the left and right boundaries because the bulk is a uniquely gapped state represented by an MPS (4.13). Indeed, if we choose a basis of the local Hilbert space on an edge $e$ as $\left\{\left|v^{i}\right\rangle_{e} \otimes\left|v_{j}\right\rangle_{e} \in M^{*} \otimes M\right\}$, we can write the ground states of the commuting projector Hamiltonian (4.21) on an interval as $\left|v^{i}\right\rangle_{1} \otimes|\Omega\rangle_{1,2} \otimes|\Omega\rangle_{2,3} \otimes \cdots \otimes|\Omega\rangle_{N-1, N} \otimes\left|v_{j}\right\rangle_{N}$, where $|\Omega\rangle_{e, e+1}:=\sum_{k}\left|v_{k}\right\rangle_{e} \otimes\left|v^{k}\right\rangle_{e+1}$ is the maximally entangled state. This expression indicates that the degrees of freedom of $M^{*}$ and $M$ remain on the left and right boundaries respectively. Therefore, the edge modes of the Hamiltonian (4.21) for a $\operatorname{Rep}(H)$ SPT phase ${ }_{K} \mathcal{M} \cong\left({ }_{H} \mathcal{M}\right)_{A}$ are described by a right $K$-module $M^{*}$ and a left $K$-module $M$, where $K=A^{\mathrm{op}} \# H^{\mathrm{cop}}$.

It is instructive to consider the case of an ordinary finite group symmetry $G$. A finite group symmetry $G$ is described by the category $\operatorname{Vec}_{G}$ of $G$-graded vector spaces, which is equivalent to the representation category of a dual group algebra $\mathbb{C}[G]^{*}$. SPT phases with this symmetry are classified by the second group cohomology $H^{2}(G, \mathrm{U}(1))[74,78,82-87]$. An SPT phase labeled by $\omega \in H^{2}(G, \mathrm{U}(1))$ is realized by the commuting projector Hamiltonian (4.21) when $A$ is a twisted group algebra $\mathbb{C}[G]^{\omega}$. The edge modes $M^{*}$ of this model become a right $\left(\mathbb{C}[G]^{\omega}\right)^{\text {op }} \#\left(\mathbb{C}[G]^{*}\right)^{\text {cop }}$-module, which is a left $\mathbb{C}[G]^{\omega}$-module in particular. This implies that these edge modes have an anomaly $\omega$ of the finite group symmetry $G$.

\subsection{Generalization to anomalous fusion category symmetries}

The most general unitary fusion category, which may or may not be anomalous, is equivalent to the representation category $\operatorname{Rep}(H)$ of a finite dimensional semisimple pseudounitary connected weak Hopf algebra $H$ [73, 88-90]. As the case of Hopf algebras, any semisimple indecomposable module category over $\operatorname{Rep}(H)$ is given by the category ${ }_{K} \mathcal{M}$ of left $K$-modules, where $K$ is an $H$-simple left $H$-comodule algebra [91]. We note that an $H$-simple left $H$-comodule algebra is semisimple [90, 91]. Accordingly, we can construct all the TQFTs ${ }_{K} \mathcal{M}$ with anomalous fusion category symmetry $\operatorname{Rep}(H)$ by pulling back the state sum TQFT with the input $K$ by a tensor functor $F_{K}: \operatorname{Rep}(H) \rightarrow{ }_{K} \mathcal{M}_{K}$. Moreover, the fact that $K$ is semisimple allows us to write down a commuting projector Hamiltonian in the same way as eq. (4.21). We can also define the action of $\operatorname{Rep}(H)$ on the lattice Hilbert space just by replacing a Hopf algebra with a weak Hopf algebra in (4.24). One

\footnotetext{
${ }^{21}$ Generally, the ground states of the Hamiltonian (4.21) on a circle are given by the center of $K$ [60]. In particular, when $K$ is simple, the ground state is unique because the center of $K$ is one-dimensional. For example, the complete Higgs phase discussed in section 4.4 is an SPT phase with $\operatorname{Rep}(G)$ symmetry.
} 
may expect that these Hamiltonians realize all the gapped phases with anomalous fusion category symmetries. However, since our proof of the commutativity of the $\operatorname{Rep}(H)$ action (4.24) and the commuting projector Hamiltonian (4.21) relies on the properties that are specific to a semisimple Hopf algebra, our proof does not work when $H$ is not a Hopf algebra, i.e. when the fusion category symmetry is anomalous. Therefore, we need to come up with another proof that is applicable to anomalous fusion category symmetries. We leave this problem to future work.

Note added. While this work was nearing completion, I became aware of a related paper by T.-C. Huang, Y.-H. Lin, and S. Seifnashri [25], in which correlation functions of 2d TQFTs with general fusion category symmetries are determined. Our result provides lattice construction of these data.

\section{Acknowledgments}

I would like to thank Masaki Oshikawa and Ken Shiozaki for comments on the manuscript. I also appreciate helpful discussions in the workshop "Topological Phase and Quantum Anomaly 2021" (YITP-T-21-03) at Yukawa Institute for Theoretical Physics, Kyoto University. I am supported by FoPM, WINGS Program, the University of Tokyo.

\section{A State sum TQFTs on surfaces with interfaces}

In this appendix, we extend the state sum TQFTs to surfaces with interfaces following [65]. A TQFT on each region separated by interfaces is described by the state sum TQFT with non-anomalous fusion category symmetry, which we defined in section 4.2 . We denote the state sum TQFT with the input $K$ as ${ }_{K} \mathcal{M}$, whose ${ }_{K} \mathcal{M}_{K}$ symmetry is pulled back to $\operatorname{Rep}(H)$ by a tensor functor $F_{K}: \operatorname{Rep}(H) \rightarrow{ }_{K} \mathcal{M}_{K}$. An interface between a $\operatorname{Rep}(H)$ symmetric TQFT ${ }_{K} \mathcal{M}$ and a $\operatorname{Rep}\left(H^{\prime}\right)$ symmetric TQFT ${ }_{K^{\prime}} \mathcal{M}$ is labeled by a $K-K^{\prime}$ bimodule $M \in{ }_{K} \mathcal{M}_{K^{\prime}}$. The possible configurations of topological defects near an interface can be classified into the following cases:

(I)

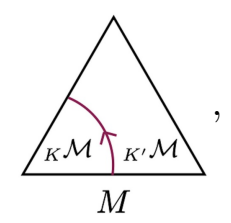

(II)

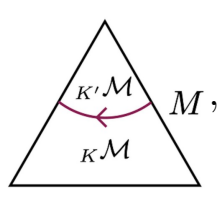

(III)

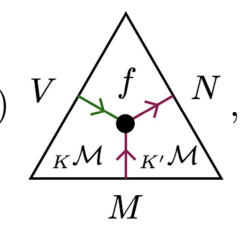

(IV)

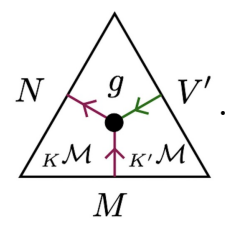

(I) and (II) represent an isolated interface $M$ between the state sum TQFTs ${ }_{K} \mathcal{M}$ and ${ }_{K^{\prime}} \mathcal{M}$. (III) and (IV) represent topological defects $V \in \operatorname{Rep}(H)$ and $V^{\prime} \in \operatorname{Rep}\left(H^{\prime}\right)$ that end on an interface. The endpoints are labeled by $K-K^{\prime}$ bimodule maps $f \in \operatorname{Hom}_{K K^{\prime}}(V \bar{\otimes} M, N)=$ $\operatorname{Hom}_{K K^{\prime}}\left(F_{K}(V) \otimes_{K} M, N\right)$ and $g \in \operatorname{Hom}_{K K^{\prime}}\left(M \bar{\otimes}^{\prime} V^{\prime}, N\right)=\operatorname{Hom}_{K K^{\prime}}\left(M \otimes_{K^{\prime}} F_{K^{\prime}}\left(V^{\prime}\right), N\right)$, where $\bar{\otimes}$ and $\bar{\otimes}^{\prime}$ are the left $\operatorname{Rep}(H)$ action and the right $\operatorname{Rep}\left(H^{\prime}\right)$ action on ${ }_{K} \mathcal{M}_{K^{\prime}}$. We 
can also consider junctions of interfaces:

$(\mathrm{V})$

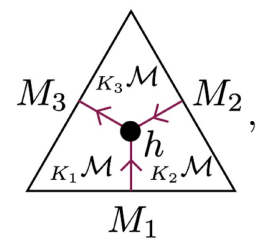

(VI)

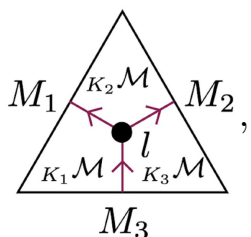

These junctions are labeled by $K_{1}-K_{3}$ bimodule maps $h \in \operatorname{Hom}_{K_{1} K_{3}}\left(M_{1} \otimes_{K_{2}} M_{2}, M_{3}\right)$ and $l \in \operatorname{Hom}_{K_{1} K_{3}}\left(M_{3}, M_{1} \otimes_{K_{2}} M_{2}\right)$, where $M_{1} \in K_{1} \mathcal{M}_{K_{2}}, M_{2} \in K_{2} \mathcal{M}_{K_{3}}$, and $M_{3} \in K_{K_{1}} \mathcal{M}_{K_{3}}$.

To incorporate these configurations, we need to extend the assignment of the vector spaces and the linear maps (4.16)-(4.19). Specifically, we add the following vector spaces and linear maps:

$R_{e}:=M \quad$ when $e$ intersects an interface $M$.

$$
Q_{(p, e)}:= \begin{cases}M & \text { when } M \text { goes into } p \text { across } e, \\ M^{*} & \text { when } M \text { goes out of } p \text { across } e .\end{cases}
$$

$$
P_{e}:=\operatorname{coev}_{M}: \mathbb{C} \rightarrow M \otimes M^{*} \quad \text { when } e \text { intersects } M \text {. }
$$

$$
E_{p}:= \begin{cases}(\mathrm{I}) & \operatorname{ev}_{M} \circ\left(\operatorname{id}_{M^{*}} \otimes \rho_{M}^{R}\right): M^{*} \otimes M \otimes K^{\prime} \rightarrow \mathbb{C}, \\ (\mathrm{II}) & \operatorname{ev}_{M} \circ\left(\operatorname{id}_{M^{*}} \otimes \rho_{M}^{L}\right): M^{*} \otimes K \otimes M \rightarrow \mathbb{C}, \\ (\mathrm{III}) & \operatorname{ev}_{N} \circ\left(\operatorname{id}_{N^{*}} \otimes\left(f \circ \pi_{F_{K}(V), M}\right)\right): N^{*} \otimes F_{K}(V) \otimes M \rightarrow \mathbb{C}, \\ (\mathrm{IV}) & \operatorname{ev}_{N} \circ\left(\operatorname{id}_{N^{*}} \otimes\left(g \circ \pi_{M, F_{K^{\prime}}\left(V^{\prime}\right)}\right)\right): N^{*} \otimes M \otimes F_{K^{\prime}}\left(V^{\prime}\right) \rightarrow \mathbb{C}, \\ (\mathrm{V}) & \operatorname{ev}_{M_{3}} \circ\left(\operatorname{id}_{M_{3}^{*}} \otimes\left(h \circ \pi_{M_{1}, M_{2}}\right)\right): M_{3}^{*} \otimes M_{1} \otimes M_{2} \rightarrow \mathbb{C}, \\ (\mathrm{VI}) & \operatorname{ev}_{M_{1} \otimes M_{2}} \circ\left(\operatorname{id}_{\left(M_{1} \otimes M_{2}\right)^{*}} \otimes\left(\iota_{M_{1}, M_{2}} \circ l\right)\right):\left(M_{1} \otimes M_{2}\right)^{*} \otimes M_{3} \rightarrow \mathbb{C} .\end{cases}
$$

The above equations (A.3)-(A.6) combined with eqs. (4.16)-(4.19) give rise to a state sum TQFT on surfaces with interfaces.

Open Access. This article is distributed under the terms of the Creative Commons Attribution License (CC-BY 4.0), which permits any use, distribution and reproduction in any medium, provided the original author(s) and source are credited.

\section{References}

[1] D. Gaiotto, A. Kapustin, N. Seiberg and B. Willett, Generalized global symmetries, JHEP 02 (2015) 172 [arXiv: 1412.5148] [INSPIRE].

[2] T. Rudelius and S.-H. Shao, Topological operators and completeness of spectrum in discrete gauge theories, JHEP 12 (2020) 172 [arXiv:2006.10052] [INSPIRE].

[3] B. Heidenreich, J. McNamara, M. Montero, M. Reece, T. Rudelius and I. Valenzuela, Non-invertible global symmetries and completeness of the spectrum, JHEP 09 (2021) 203 [arXiv: 2104.07036] [INSPIRE].

[4] T. Johnson-Freyd, $(3+1) D$ topological orders with only a $Z_{2}$-charged particle, arXiv:2011.11165 [INSPIRE]. 
[5] T. Johnson-Freyd and M. Yu, Topological orders in (4+1)-dimensions, arXiv: 2104.04534 [INSPIRE].

[6] L. Kong, Y. Tian and Z.-H. Zhang, Defects in the 3-dimensional toric code model form a braided fusion 2-category, JHEP 12 (2020) 078 [arXiv: 2009. 06564] [INSPIRE].

[7] M. Koide, Y. Nagoya and S. Yamaguchi, Non-invertible topological defects in 4-dimensional $Z_{2}$ pure lattice gauge theory, PTEP 2022 (2022) 013B03 [arXiv:2109.05992] [INSPIRE].

[8] J. Fröhlich, J. Fuchs, I. Runkel and C. Schweigert, Defect lines, dualities, and generalised orbifolds, in $16^{\text {th }}$ international congress on mathematical physics, World Scientific, Singapore (2009) [arXiv:0909.5013] [INSPIRE].

[9] I. Brunner, N. Carqueville and D. Plencner, Orbifolds and topological defects, Commun. Math. Phys. 332 (2014) 669 [arXiv: 1307.3141] [InSPIRE].

[10] I. Brunner, N. Carqueville and D. Plencner, A quick guide to defect orbifolds, Proc. Symp. Pure Math. 88 (2014) 231 [arXiv:1310.0062] [INSPIRE].

[11] I. Brunner, N. Carqueville and D. Plencner, Discrete torsion defects, Commun. Math. Phys. 337 (2015) 429 [arXiv:1404.7497] [INSPIRE].

[12] N. Carqueville and I. Runkel, Orbifold completion of defect bicategories, Quantum Topol. 7 (2016) 203 [arXiv: 1210.6363] [INSPIRE].

[13] L. Bhardwaj and Y. Tachikawa, On finite symmetries and their gauging in two dimensions, JHEP 03 (2018) 189 [arXiv:1704.02330] [INSPIRE].

[14] C.-M. Chang, Y.-H. Lin, S.-H. Shao, Y. Wang and X. Yin, Topological defect lines and renormalization group flows in two dimensions, JHEP 01 (2019) 026 [arXiv:1802.04445] [INSPIRE].

[15] R. Thorngren and Y. Wang, Fusion category symmetry I: anomaly in-flow and gapped phases, arXiv:1912.02817 [INSPIRE].

[16] W. Ji, S.-H. Shao and X.-G. Wen, Topological transition on the conformal manifold, Phys. Rev. Res. 2 (2020) 033317 [arXiv: 1909.01425] [InSPIRE].

[17] Y.-H. Lin and S.-H. Shao, Duality defect of the monster CFT, J. Phys. A 54 (2021) 065201 [arXiv: 1911.00042] [INSPIRE].

[18] T. Lichtman, R. Thorngren, N.H. Lindner, A. Stern and E. Berg, Bulk anyons as edge symmetries: boundary phase diagrams of topologically ordered states, Phys. Rev. B 104 (2021) 075141 [arXiv: 2003.04328] [INSPIRE].

[19] Z. Komargodski, K. Ohmori, K. Roumpedakis and S. Seifnashri, Symmetries and strings of adjoint $Q C D_{2}$, JHEP 03 (2021) 103 [arXiv:2008.07567] [INSPIRE].

[20] C.-M. Chang and Y.-H. Lin, Lorentzian dynamics and factorization beyond rationality, JHEP 10 (2021) 125 [arXiv:2012.01429] [INSPIRE].

[21] T.-C. Huang and Y.-H. Lin, Topological field theory with Haagerup symmetry, arXiv:2102.05664 [INSPIRE].

[22] K. Inamura, Topological field theories and symmetry protected topological phases with fusion category symmetries, JHEP 05 (2021) 204 [arXiv:2103.15588] [INSPIRE].

[23] R. Thorngren and Y. Wang, Fusion category symmetry II: categoriosities at $c=1$ and beyond, arXiv:2106.12577 [INSPIRE]. 
[24] T.-C. Huang, Y.-H. Lin, K. Ohmori, Y. Tachikawa and M. Tezuka, Numerical evidence for a Haagerup conformal field theory, arXiv:2110.03008 [INSPIRE].

[25] T.-C. Huang, Y.-H. Lin and S. Seifnashri, Construction of two-dimensional topological field theories with non-invertible symmetries, JHEP 12 (2021) 028 [arXiv:2110.02958] [INSPIRE].

[26] R. Vanhove et al., A critical lattice model for a Haagerup conformal field theory, arXiv:2110.03532 [INSPIRE].

[27] D. Aasen, R.S.K. Mong and P. Fendley, Topological defects on the lattice I: the Ising model, J. Phys. A 49 (2016) 354001 [arXiv:1601.07185] [InSPIRE].

[28] D. Aasen, P. Fendley and R.S.K. Mong, Topological defects on the lattice: dualities and degeneracies, arXiv:2008.08598 [INSPIRE].

[29] A. Feiguin et al., Interacting anyons in topological quantum liquids: the golden chain, Phys. Rev. Lett. 98 (2007) 160409 [cond-mat/0612341] [INSPIRE].

[30] C. Gils, E. Ardonne, S. Trebst, A.W.W. Ludwig, M. Troyer and Z. Wang, Collective states of interacting anyons, edge states, and the nucleation of topological liquids, Phys. Rev. Lett. 103 (2009) 070401 [arXiv:0810.2277].

[31] M. Buican and A. Gromov, Anyonic chains, topological defects, and conformal field theory, Commun. Math. Phys. 356 (2017) 1017 [arXiv:1701.02800] [INSPIRE].

[32] M. Nguyen, Y. Tanizaki and M. Ünsal, Semi-Abelian gauge theories, non-invertible symmetries, and string tensions beyond $N$-ality, JHEP 03 (2021) 238 [arXiv:2101.02227] [INSPIRE].

[33] M. Nguyen, Y. Tanizaki and M. Ünsal, Noninvertible 1-form symmetry and Casimir scaling in $2 D$ Yang-Mills theory, Phys. Rev. D 104 (2021) 065003 [arXiv:2104.01824] [InSPIRE].

[34] E. Sharpe, Topological operators, noninvertible symmetries and decomposition, arXiv:2108.13423 [INSPIRE].

[35] N. Carqueville, C. Meusburger and G. Schaumann, 3-dimensional defect TQFTs and their tricategories, Adv. Math. 364 (2020) 107024 [arXiv: 1603.01171] [INSPIRE].

[36] N. Carqueville, I. Runkel and G. Schaumann, Line and surface defects in Reshetikhin-Turaev TQFT, Quant. Topol. 10 (2018) 399 [arXiv:1710.10214] [INSPIRE].

[37] N. Carqueville, I. Runkel and G. Schaumann, Orbifolds of n-dimensional defect TQFTs, Geom. Topol. 23 (2019) 781 [arXiv:1705.06085] [INSPIRE].

[38] N. Carqueville, I. Runkel and G. Schaumann, Orbifolds of Reshetikhin-Turaev TQFTs, Theor. Appl. Categor. 35 (2020) 513 [arXiv:1809.01483] [INSPIRE].

[39] W. Ji and X.-G. Wen, Categorical symmetry and noninvertible anomaly in symmetry-breaking and topological phase transitions, Phys. Rev. Res. 2 (2020) 033417 [arXiv: 1912.13492] [INSPIRE].

[40] T. Johnson-Freyd, On the classification of topological orders, arXiv:2003.06663 [INSPIRE].

[41] L. Kong and H. Zheng, Categories of quantum liquids I, arXiv:2011.02859 [INSPIRE].

[42] L. Kong, T. Lan, X.-G. Wen, Z.-H. Zhang and H. Zheng, Algebraic higher symmetry and categorical symmetry - a holographic and entanglement view of symmetry, Phys. Rev. Res. 2 (2020) 043086 [arXiv:2005.14178] [INSPIRE]. 
[43] A. Kapustin, Topological field theory, higher categories, and their applications, in International congress of mathematicians, (2010) [arXiv:1004.2307] [INSPIRE].

[44] C.L. Douglas and D.J. Reutter, Fusion 2-categories and a state-sum invariant for 4-manifolds, arXiv:1812.11933.

[45] H.A. Kramers and G.H. Wannier, Statistics of the two-dimensional ferromagnet. Part 1, Phys. Rev. 60 (1941) 252 [inSPIRE].

[46] J. Fröhlich, J. Fuchs, I. Runkel and C. Schweigert, Kramers-Wannier duality from conformal defects, Phys. Rev. Lett. 93 (2004) 070601 [cond-mat/0404051] [inSPIRE].

[47] D. Tambara and S. Yamagami, Tensor categories with fusion rules of self-duality for finite Abelian groups, J. Alg. 209 (1998) 692.

[48] E.P. Verlinde, Fusion rules and modular transformations in $2 D$ conformal field theory, Nucl. Phys. B 300 (1988) 360 [INSPIRE].

[49] J. Fuchs, M.R. Gaberdiel, I. Runkel and C. Schweigert, Topological defects for the free boson CFT, J. Phys. A 40 (2007) 11403 [arXiv:0705.3129] [inSPIRE].

[50] V.B. Petkova and J.-B. Zuber, Generalized twisted partition functions, Phys. Lett. B 504 (2001) 157 [hep-th/0011021] [INSPIRE].

[51] J. Fröhlich, J. Fuchs, I. Runkel and C. Schweigert, Duality and defects in rational conformal field theory, Nucl. Phys. B 763 (2007) 354 [hep-th/0607247] [INSPIRE].

[52] J. Fuchs, I. Runkel and C. Schweigert, TFT construction of RCFT correlators. 1. Partition functions, Nucl. Phys. B 646 (2002) 353 [hep-th/0204148] [INSPIRE].

[53] J. Fuchs, I. Runkel and C. Schweigert, TFT construction of RCFT correlators. 2. Unoriented world sheets, Nucl. Phys. B 678 (2004) 511 [hep-th/0306164] [InSPIRE].

[54] J. Fuchs, I. Runkel and C. Schweigert, TFT construction of RCFT correlators. 3. Simple currents, Nucl. Phys. B 694 (2004) 277 [hep-th/0403157] [INSPIRE].

[55] J. Fuchs, I. Runkel and C. Schweigert, TFT construction of RCFT correlators. 4. Structure constants and correlation functions, Nucl. Phys. B 715 (2005) 539 [hep-th/0412290] [INSPIRE].

[56] J. Fjelstad, J. Fuchs, I. Runkel and C. Schweigert, TFT construction of RCFT correlators. 5. Proof of modular invariance and factorisation, Theor. Appl. Categor. 16 (2006) 342 [hep-th/0503194] [INSPIRE].

[57] J. Wang, X.-G. Wen and E. Witten, Symmetric gapped interfaces of SPT and SET states: systematic constructions, Phys. Rev. X 8 (2018) 031048 [arXiv: 1705.06728] [INSPIRE].

[58] N. Andruskiewitsch and J.M. Mombelli, On module categories over finite-dimensional Hopf algebras, J. Alg. 314 (2007) 383 [math.QA/0608781].

[59] C.E. Watts, Intrinsic characterizations of some additive functors, Proc. Amer. Math. Soc. 11 (1960) 5 .

[60] M. Fukuma, S. Hosono and H. Kawai, Lattice topological field theory in two-dimensions, Commun. Math. Phys. 161 (1994) 157 [hep-th/9212154] [INSPIRE].

[61] S. Skryabin, Projectivity and freeness over comodule algebras, Trans. Amer. Math. Soc. 359 (2007) 2597 [math.RA/0610657]. 
[62] D.J. Williamson and Z. Wang, Hamiltonian models for topological phases of matter in three spatial dimensions, Annals Phys. 377 (2017) 311 [arXiv:1606.07144] [INSPIRE].

[63] A.L. Bullivant, Exactly solvable models for topological phases of matter and emergent excitations, Ph.D. thesis, U. Leeds, Leeds, U.K. (2018).

[64] A.D. Lauda and H. Pfeiffer, State sum construction of two-dimensional open-closed topological quantum field theories, J. Knot Theor. Ramifications 16 (2007) 1121 [math.QA/0602047] [INSPIRE].

[65] A. Davydov, L. Kong and I. Runkel, Field theories with defects and the centre functor, in Mathematical foundations of quantum field theory and perturbative string theory, $\mathrm{H}$. Sati and U. Schreiber eds., Proc. Symp. Pure Math. 83, American Mathematical Society, Providence, RI, U.S.A. (2011), pg. 71 [arXiv:1107.0495] [INSPIRE].

[66] P. Etingof, S. Gelaki, D. Nikshych and V. Ostrik, Tensor categories, Math. Surv. Monogr. 205 (2015) 1.

[67] J. Fuchs and C. Stigner, On Frobenius algebras in rigid monoidal categories, Arabian J. Sci. Eng. 33-2C (2009) 175 [arXiv:0901.4886].

[68] S. Montgomery, Hopf algebras and their actions on rings, CBMS Regional Conf. Ser. Math. 82 (1993) 1.

[69] S. Montgomery, Representation theory of semisimple Hopf algebras, in Algebra representation theory. Proceedings of the NATO Advanced Study Institute, Constanta, Romania, 2-12 August 2000, Kluwer Academic Publishers, Dordrecht, The Netherlands (2001), pg. 189.

[70] H.--J. Schneider, Lectures on Hopf algebras. Notes by Sonia Natale, Facultad de Matemática, Astronomía y Física, Universidad Nacional de Córdoba, Córdoba, Argentina (1995).

[71] T. Banica and J. Bichon, Hopf images and inner faithful representations, Glasgow Math. J. 52 (2010) 677 [arXiv:0807.3827].

[72] P. Etingof and V. Ostrik, Finite tensor categories, Moscow Math. J. 4 (2004) 627 [math.QA/0301027].

[73] V. Ostrik, Module categories, weak Hopf algebras and modular invariants, Transf. Groups 8 (2003) 177 [math.QA/0111139].

[74] G.W. Moore and G. Segal, D-branes and k-theory in $2 D$ topological field theory, hep-th/0609042 [INSPIRE].

[75] A. Kapustin, A. Turzillo and M. You, Topological field theory and matrix product states, Phys. Rev. B 96 (2017) 075125 [arXiv: 1607.06766] [INSPIRE].

[76] M. Yan and Y. Zhu, Stabilizer for Hopf algebra actions, Commun. Alg. 26 (1998) 3885.

[77] K. Shiozaki and S. Ryu, Matrix product states and equivariant topological field theories for bosonic symmetry-protected topological phases in $(1+1)$ dimensions, JHEP 04 (2017) 100 [arXiv: 1607.06504] [INSPIRE].

[78] V. Turaev, Homotopy field theory in dimension two and group algebras, math.QA/9910010 [INSPIRE].

[79] D. Harlow and H. Ooguri, Symmetries in quantum field theory and quantum gravity, Commun. Math. Phys. 383 (2021) 1669 [arXiv: 1810.05338] [INSPIRE]. 
[80] C. Vafa, Modular invariance and discrete torsion on orbifolds, Nucl. Phys. B 273 (1986) 592 [INSPIRE].

[81] R. Dijkgraaf and E. Witten, Topological gauge theories and group cohomology, Commun. Math. Phys. 129 (1990) 393 [inSPIRE].

[82] X. Chen, Z.-C. Gu and X.-G. Wen, Classification of gapped symmetric phases in one-dimensional spin systems, Phys. Rev. B 83 (2011) 035107 [arXiv:1008.3745].

[83] X. Chen, Z.-C. Gu and X.-G. Wen, Complete classification of one-dimensional gapped quantum phases in interacting spin systems, Phys. Rev. B 84 (2011) 235128 [arXiv: 1103.3323].

[84] L. Fidkowski and A. Kitaev, Topological phases of fermions in one dimension, Phys. Rev. B 83 (2011) 075103 [arXiv: 1008.4138].

[85] N. Schuch, D. Pérez-García and I. Cirac, Classifying quantum phases using matrix product states and projected entangled pair states, Phys. Rev. B 84 (2011) 165139 [arXiv: 1010.3732].

[86] X. Chen, Z.-C. Gu, Z.-X. Liu and X.-G. Wen, Symmetry protected topological orders and the group cohomology of their symmetry group, Phys. Rev. B 87 (2013) 155114 [arXiv: 1106.4772] [INSPIRE].

[87] A. Kapustin and A. Turzillo, Equivariant topological quantum field theory and symmetry protected topological phases, JHEP 03 (2017) 006 [arXiv: 1504.01830] [INSPIRE].

[88] D. Nikshych, V. Turaev and L. Vainerman, Invariants of knots and 3-manifolds from quantum groupoids, Topology Appl. 127 (2003) 91 [math.QA/0006078].

[89] T. Hayashi, A canonical Tannaka duality for finite seimisimple tensor categories, math.QA/9904073.

[90] D. Nikshych, Semisimple weak Hopf algebras, J. Alg. 275 (2004) 639 [math.QA/0304098].

[91] H. Henker, Module categories over quasi-Hopf algebras and weak Hopf algebras and the projectivity of Hopf modules, Ph.D. thesis, Ludwig-Maximilians-Universität München, Munich, Germany May 2011. 